\title{
Vortex bursting and tracer transport of a counter-rotating vortex pair
}

\author{
T. Misaka, ${ }^{1, a)}$ F. Holzäpfel, ${ }^{1}$ I. Hennemann, ${ }^{1}$ T. Gerz, ${ }^{1}$ \\ M. Manhart, ${ }^{2}$ and F. Schwertfirm ${ }^{3}$ \\ ${ }^{1}$ Deutsches Zentrum für Luft- und Raumfahrt (DLR), Institut für Physik der Atmosphäre, \\ 82234 Oberpfaffenhofen, Germany \\ ${ }^{2}$ Technische Universität München, Fachgebiet Hydromechanik, 80333 München, Germany \\ ${ }^{3}$ Kreuzinger+Manhart Turbulenz GmbH, 81765 München, Germany
}

(Received 19 April 2011; accepted 21 December 2011; published online 27 February 2012)

\begin{abstract}
Large-eddy simulations of a coherent counter-rotating vortex pair in different environments are performed. The environmental background is characterized by varying turbulence intensities and stable temperature stratifications. Turbulent exchange processes between the vortices, the vortex oval, and the environment, as well as the material redistribution processes along the vortex tubes are investigated employing passive tracers that are superimposed to the initial vortex flow field. It is revealed that the vortex bursting phenomenon, known from photos of aircraft contrails or smoke visualization, is caused by collisions of secondary vortical structures traveling along the vortex tube which expel material from the vortex but do not result in a sudden decay of circulation or an abrupt change of vortex core structure. In neutrally stratified and weakly turbulent conditions, vortex reconnection triggers traveling helical vorticity structures which is followed by their collision. A long-lived vortex ring links once again establishing stable double rings. Key phenomena observed in the simulations are supported by photographs of contrails. The vertical and lateral extents of the detrained passive tracer strongly depend on environmental conditions where the sensitivity of detrainment rates on initial tracer distributions appears to be low. (c) 2012 American Institute of Physics. [http://dx.doi.org/10.1063/1.3684990]
\end{abstract}

\section{INTRODUCTION}

The dynamics of a pair of counter-rotating vortices in various background conditions has been a subject of numerous studies over decades. The incentive to study this particular flow has been motivated by the potential risk of the wake vortex pairs generated behind flying aircraft posed to following aircraft. Comprehensive and detailed understandings of wake vortex behavior under different atmospheric conditions are crucial for the development of wake vortex prediction systems that aim to increase aviation safety and airport capacity. ${ }^{1}$ But this type of flow is also of fundamental interest to fluid dynamicists as aircraft wake vortices constitute a relatively simple configuration of coherent vortex structures which are embedded in incoherent, i.e., turbulent and stratified environments. The study of such flows-experimentally as well as numerically_yields improved understanding of elementary vortex interaction that is also relevant in more complex transitional and turbulent flows. On the other hand, turbulent mixing processes and detrainment of the ice crystals generated from the exhaust jets during wake vortex descent may affect the generation of condensation trails (contrails). The contrails in turn may trigger the formation of long-lived cirrus clouds (contrail cirrus) that have been suspected to contribute to global warming by modifying the radiation budget of the atmosphere. ${ }^{2,3}$ The relevance of these topics might become increasingly large due to the expected increase of air traffic in the coming decades. ${ }^{4}$

\footnotetext{
a)Electronic mail: Takashi.Misaka@dlr.de. 
The wake vortex phenomenon in the atmosphere is related to a wide range of flows with scales ranging from few millimeters to several tens of kilometers. In particular, the flows around the aircraft's main wings, slats, flaps, and their interactions may affect wake vortex roll-up in high-lift conditions. ${ }^{5}$ On the other hand, wake vortices generated by cruising aircraft may remain along several tens of kilometers. ${ }^{2}$ The transport and mixing of aircraft emissions can be divided into four regimes: ${ }^{6}$ (1) jet regime, (2) vortex regime, (3) dissipation regime, and (4) diffusion regime. Numerical simulation setups are typically limited to flow scales of one or two of these individual regimes. Dynamics of wake vortices in the vortex and dissipation regime have been studied mainly by largeeddy simulation (LES) or direct numerical simulation. In these simulations, the detailed temporal evolution of a vortex pair with an initially longitudinally constant velocity profile is investigated allowing for the formation of cooperative instabilities, e.g., short-wave (elliptic) instability ${ }^{7-10}$ and Crow instability. ${ }^{11-14}$ Various atmospheric conditions of turbulence, stability, and wind shear have been considered in order to assess the influence of these factors on wake vortex evolution and decay. ${ }^{15,16}$ However, little is known about the further evolution of the vortex rings formed as a consequence of the Crow instability in particular under different environmental conditions.

The vortex bursting phenomenon has been reported from flight experiments visualized by smoke ${ }^{17,18}$ or contrails, ${ }^{19}$ in the towing tank ${ }^{20}$ and numerical simulations. ${ }^{21}$ The details of the descriptions differ but they are always connected with an abrupt change of the diameter of the marker around the vortex core. It has been observed that the bursts travel along the vortex tube in either direction. Sometimes two bursts would travel toward each other, eventually colliding and leaving behind an intensely marked disk-like parcel of tracer, ${ }^{18}$ which is also termed puff or pancake. ${ }^{22}$ In the vortex core region, funnel-shaped features have been observed surrounded by the pancake-shaped structures. ${ }^{19}$ No explanation of the causes and structure of the bursts has been offered until Moet et al. ${ }^{21}$ suggested that vortex bursting might be caused by the collision of helical vorticity structures connected with pressure waves emanating from the location of vortex reconnection. We confirm that the colliding helical vorticity structures indeed temporarily and locally reduce circulation, but do not cause substantial radial spreading of the tracer. Instead, we suggest that vortex bursting associated with radial spreading of tracer before the onset of vortex linking is caused by the collision of propagating secondary vortical structures which do not cause a change of vortex core structure.

For numerical simulations of contrails, microphysics of the ice particles have been studied along with wake vortex dynamics. ${ }^{23-26}$ The consideration of microphysics is crucial for obtaining realistic optical depths and coverage of contrails which is required to estimate radiation effects. On the other hand, our focus on passive tracers enables to investigate turbulent transport and mixing processes precluding uncertainties regarding the performance of the microphysics package and adulteration caused by sublimation or sedimentation of ice particles. Nevertheless, it is still possible to compare the simulated passive tracer distributions with wake vortices visualized by smoke experiments ${ }^{17,20,27}$ or contrails under certain conditions.

With this LES study, we aim to explain some details of the decay of coherent vortices in stably stratified and weakly turbulent background flows. The parameter setting represents conditions of the atmosphere where large civil aviation aircraft typically cruise. By this we also shed light on tracer distributions as observed along the vortices and tracer detrainment into the atmosphere. The impact of coherent-incoherent and coherent-coherent vortex interactions on vortex decay and tracer distribution and detrainment is discussed. In the following Secs. II, III, and IV, applied numerical methodologies, the initialization of wake vortices with various cross-sectional distributions of the passive tracers, and the generation of stably stratified ambient turbulence fields are respectively described. In Sec. V, the results on characteristics of a vortex pair in the different environments: secondary vortical structures, tracer redistribution, and detrainment mechanisms are presented and discussed. Sec. VI concludes our study.

\section{EQUATIONS AND NUMERICAL METHODS}

The LES are conducted using MGLET, which is a finite volume solver for the incompressible Navier-Stokes equations. ${ }^{28}$ To enable wake vortex simulations in stably stratified atmospheric conditions featuring buoyancy effects, an additional equation is solved for potential temperature, 
employing the Boussinesq approximation ${ }^{29}$

$$
\begin{gathered}
\frac{\partial u_{i}}{\partial t}+\frac{\partial\left(u_{i} u_{j}\right)}{\partial x_{j}}=-\frac{1}{\rho_{0}} \frac{\partial p^{\prime}}{\partial x_{i}}+\left(v+v_{t}\right) \frac{\partial^{2} u_{i}}{\partial x_{j}^{2}}+g \frac{\theta^{\prime}}{\theta_{0}} \delta_{i 3}, \\
\frac{\partial \theta^{\prime}}{\partial t}+\frac{\partial\left(u_{j} \theta^{\prime}\right)}{\partial x_{j}}=\left(\kappa+\kappa_{t}\right) \frac{\partial^{2} \theta^{\prime}}{\partial x_{j}^{2}}+u_{3} \frac{d \theta_{s}}{d x_{3}}, \\
\frac{\partial u_{j}}{\partial x_{j}}=0 .
\end{gathered}
$$

In Eqs. (1)-(3), $u_{i}, p^{\prime}$, and $\theta^{\prime}$ represent the cell averaged velocity components in three spatial directions $(i, j=1,2$, or 3$)$, the cell averaged pressure and potential temperature fluctuations, respectively. The summation convention is used for the velocity components $u_{i}$ and $\delta_{i j}$ denotes Kronecker's delta. The primes for pressure and potential temperature indicate that those are defined by the deviation from the reference states: $p=p_{0}+p^{\prime}, \theta=\theta_{0}+\theta^{\prime}$. In this study, typical values of air density and potential temperature at cruise altitude are employed: $\rho_{0}=0.35 \mathrm{~kg} / \mathrm{m}^{3}$ and $\theta_{0}=332.1 \mathrm{~K}^{30,31}$ The background potential temperature $\theta_{s}$ in Eq. (2) is used to specify the vertical gradient of potential temperature and is set to constant in a neutrally stratified case. In the Boussinesq approximation, potential temperature and the momentum equations are coupled via the vertical velocity component $u_{3}$. The kinematic viscosity in Eq. (1) is defined by the sum of molecular viscosity and eddy viscosity obtained by a subgrid-scale model. The corresponding diffusion coefficients in Eq. (2) are obtained by assuming constant molecular and turbulent Prandtl numbers of 0.7 and 0.9 , respectively. In addition, equations for passive tracers are employed considering advection induced by local velocity and diffusion by molecular and turbulent viscosity.

The above equations are solved by a compact fourth-order finite volume scheme. ${ }^{32,33}$ A splitinterface algorithm is used for the parallelization of tri-diagonal systems of the compact scheme, which realizes smaller overhead time and scalability in parallel environments. ${ }^{34}$ The pressure field is obtained by the velocity-pressure iteration method by Hirt and Cook. ${ }^{35}$ Iterations are performed until the divergence of the velocity field becomes smaller than a threshold value of $1.0 \times 10^{-5} \mathrm{~s}^{-1}$. The third-order Runge-Kutta method is used for time integration. ${ }^{36}$

The Lagrangian dynamic model is employed to define eddy viscosity $v_{t}$ in Eq. (1) (Ref. 37). The use of the standard Smagorinsky model results in excessive eddy viscosity in the vortex core, hence, a correction procedure is usually used together with the standard Smagorinsky model. ${ }^{38,39}$ An alternative way to handle vortex flows is the use of a dynamic-type subgrid scale model. The Lagrangian dynamic model does not require directions of statistical homogeneity for the averaging process of subgrid model coefficients but calculates the required averages along path-lines. This enables the Lagrangian dynamic model to distinguish between the centrifugally stable vortex core regions and the external turbulent flow.

\section{INITIAL CONDITIONS}

The coherent wake vortices are initialized as a pair of counter-rotating Lamb-Oseen vortices which represents fully rolled-up trailing vortices of a cruising large aircraft. The vortices possess a circulation of $\Gamma_{0}=530.0 \mathrm{~m}^{2} / \mathrm{s}$, a vortex core radius of $r_{c}=3.0 \mathrm{~m}$, and a vortex spacing of $b_{0}=47.1 \mathrm{~m}$. The corresponding reference length, velocity, and time scales used for normalization of the results are defined by the initial vortex spacing $b_{0}$, the initial vortex descent speed $w_{0}=\Gamma_{0} /\left(2 \pi b_{0}\right)=1.79 \mathrm{~m} / \mathrm{s}$, and the time for descending one vortex spacing $t_{0}=b_{0} / w_{0}=26.3 \mathrm{~s}$, respectively. Turbulent fluctuations induced by the aircraft's jets, wings, and fuselage are modeled by white noise with a weighting of a Gaussian distribution possessing a maximum variance of $1 \mathrm{~m} / \mathrm{s}$ at the vortex core radius. ${ }^{40}$ Molecular kinematic viscosity is set to $v=4.0 \times 10^{-5} \mathrm{~m}^{2} / \mathrm{s}$ such that the vortex circulation based Reynolds number amounts to $\operatorname{Re}=\Gamma_{0} / v \approx 10^{7}$. The dimensions of the computational domain are $L_{x}=400, L_{y}=384$, and $L_{z}=512 \mathrm{~m}$ where $x, y$, and $z$ denote flight, spanwise, and vertical directions, respectively (see Fig. 1). The domain length covers a theoretical wave length of the Crow instability. ${ }^{13}$ A uniform mesh spacing of $1 \mathrm{~m}$ is employed for all three directions unless otherwise stated. 


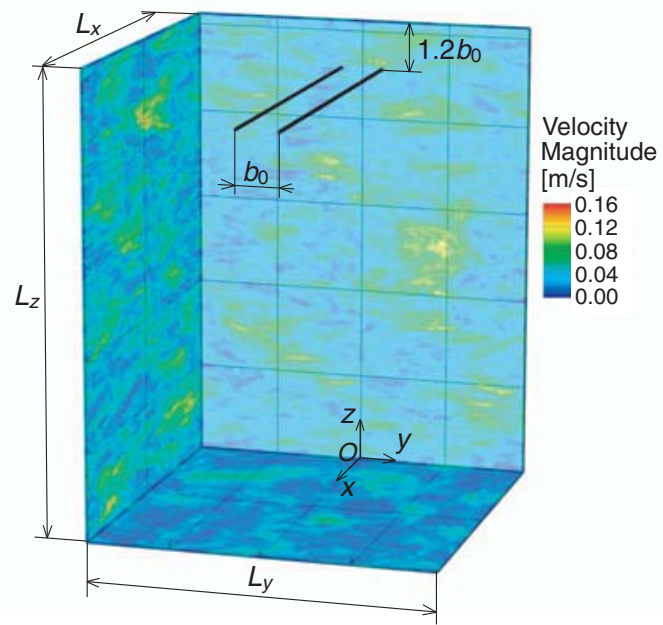

FIG. 1. (Color online) Schematic of computational domain and initial vortex position represented by thick black lines. Velocity magnitude fields established after the pre-run of a weakly turbulent and moderately stable environment are shown along the domain boundaries.

We consider three initial cross-sectional distributions of passive tracer superimposed to the wake vortices. In the two extreme cases, the tracer is trapped either in the vortex core or in the vortex oval envelope. In the more realistic case, the tracer is situated within the streamlines covering half of the vortex oval's cross-sectional area (see Fig. 2). The half-oval tracer distribution was motivated by observations of contrails after completion of the vortex roll-up. Cross-sectional areas covered by these distributions are 113,3150 , and $6300 \mathrm{~m}^{2}$ for vortex core, half-oval, and full-oval, respectively. Based on a fit obtained from observations of exhaust plume areas, ${ }^{41,42}$ the cross-sectional area of the half-oval of $3150 \mathrm{~m}^{2}$ corresponds to 38 seconds of plume age which is on the order of one vortex reference time $t_{0}$. Hence, the assumption of a fully rolled-up vortex pair and the initialized tracer distribution are consistent.

The vortex core and full-oval initializations have an internal tracer value of one and the background value of zero where a smooth transition is realized by a hyperbolic tangent function. The
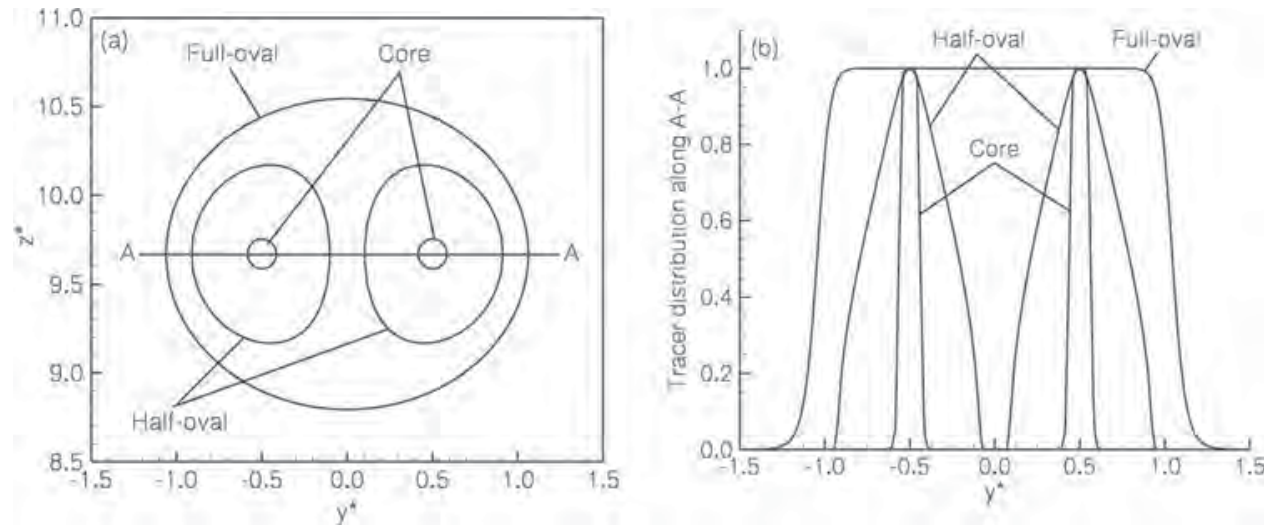

FIG. 2. Initial distributions of passive tracer: (a) cross-sectional distribution, (b) the distribution along section A-A. 
vortex core tracer distribution is given by

$$
\begin{aligned}
c_{v}(y, z) & =0.5\left[1.0-\tanh \left[2.5\left(\frac{r_{1}}{r_{c}}-\frac{r_{c}}{r_{1}}\right)\right]\right] \\
& +0.5\left[1.0-\tanh \left[2.5\left(\frac{r_{2}}{r_{c}}-\frac{r_{c}}{r_{2}}\right)\right]\right],
\end{aligned}
$$

where $r_{1}=\left[\left(y-y_{s}\right)^{2}+\left(z-z_{s}\right)^{2}\right]^{1 / 2}$ and $r_{2}=\left[\left(y-y_{p}\right)^{2}+\left(z-z_{p}\right)^{2}\right]^{1 / 2}$ are distances from the centers of the starboard $\left(y_{s}, z_{s}\right)$ and port vortex $\left(y_{p}, z_{p}\right)$, respectively. Similarly, a profile for the full-oval is defined as

$$
c_{f}(y, z)=0.5\left[1.0-\tanh \left[2.5\left(\frac{r}{r_{o}}-\frac{r_{o}}{r}\right)\right]\right],
$$

where $r_{o}=\left[\left(1.0-\left(A_{s} / A_{l}\right)^{2}\right) y^{2}+A_{s}^{2}\right]^{1 / 2},-A_{l} \leq y \leq A_{l}$ defines the vortex oval and $r=\left[\left(y-y_{o}\right)^{2}\right.$ $\left.+\left(z-z_{o}\right)^{2}\right]^{1 / 2}$ is the distance from the center of the oval $\left(y_{o}, z_{o}\right)$. Here, $A_{s}$ and $A_{l}$ are short and long axes of the oval, respectively.

The tracer concentration within the half-oval corresponds to the magnitude of the modified stream function of a vortex pair $c_{h}(y, z)=|\Psi(y, z)|$,

$$
\Psi(y, z)=\left\{\begin{array}{l}
0.761\left[\frac{y}{b_{0}}+\ln \left(\frac{\sqrt{z^{2}+\left(y-b_{0} / 2\right)^{2}}}{\sqrt{z^{2}+\left(y+b_{0} / 2\right)^{2}}}\right)-0.128\right]^{\frac{1}{2}} \\
(y>0, \Psi<-0.128 \text { or } y<0, \Psi>0.128), \\
0 \quad \text { (otherwise), }
\end{array}\right.
$$

where the constant 0.761 is a factor to normalize the maximum concentration value to one, while the constant 0.128 adjusts the area covered by the tracer to half of the full-oval's cross-sectional area.

The visualizations of vortex structures and tracer distributions in this paper are produced only from every second grid point of the computational mesh. However, the full resolution of the simulations is used for the evaluations of, e.g., vortex positions and circulation.

\section{AMBIENT TURBULENCE FIELD}

The ambient atmospheric conditions are characterized by different eddy dissipation rates $\varepsilon$, representing the strength of ambient turbulence and Brunt-Väisälä frequencies $N=\left(g / \theta_{0} d \theta / d z\right)^{1 / 2}$, representing the stability of the temperature stratification. The analysis of in situ measurements of the Falcon research aircraft ${ }^{31}$ at altitudes between 9 and $11 \mathrm{~km}$ indicates dissipation rates between $10^{-8}$ and $2 \times 10^{-7} \mathrm{~m}^{2} / \mathrm{s}^{3}$ and Brunt-Väisälä frequencies typically ranging from $0.011 \mathrm{~s}^{-1}$ to $0.023 \mathrm{~s}^{-1}$. The combinations of $\varepsilon^{*}$ and $N^{*}$ investigated in this study are listed in Table I in which $\varepsilon$ and $N$ are non-dimensionalized based on wake vortex parameters according to $\varepsilon^{*}=\left(\varepsilon b_{0}\right)^{1 / 3} / w_{0}$ and $N^{*}=N t_{0}$, respectively. Table I also provides the normalized integral turbulence length-scales, $L_{t}^{*}=L_{t} / b_{0}$, reached at the end of the respective pre-runs for generating the ambient turbulence field. It has been pointed out that the turbulence length-scale, in addition to eddy dissipation rate

TABLE I. Investigated combinations of normalized eddy dissipation rates $\varepsilon^{*}$, Brunt-Väisälä frequencies $N^{*}$, and respective integral turbulence length scales $L_{t}^{*}$.

\begin{tabular}{lccc}
\hline \hline & $N^{*}=0.0$ & 0.35 & 1.0 \\
\hline$\varepsilon^{*}=0.0$ & - & $L_{t}^{*}=\infty$ & - \\
0.01 & $L_{t}^{*}=0.85$ & $L_{t}^{*}=0.96$ & $L_{t}^{*}=0.79$ \\
0.23 & - & $L_{t}^{*}=0.72$ & - \\
\hline \hline
\end{tabular}




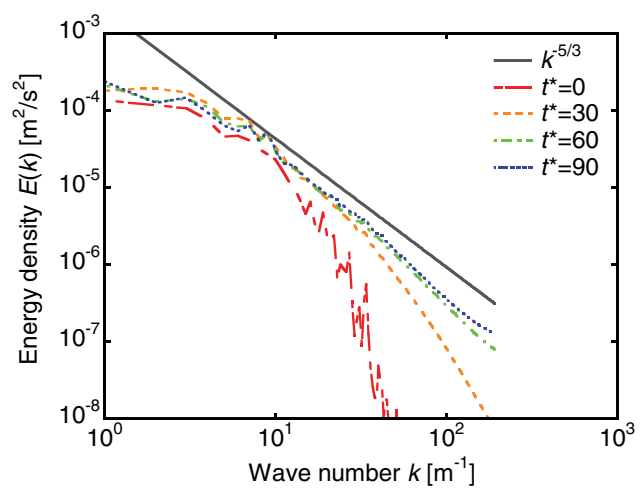

FIG. 3. (Color online) Energy density spectra established during the pre-run for the baseline case with $\varepsilon^{*}=0.01$ and $N^{*}=0.35$.

and Brunt-Väisälä frequency, influences the decay of a vortex pair. ${ }^{43}$ The baseline case with very weak turbulence $\varepsilon^{*}=0.01\left(\varepsilon=1.2 \times 10^{-7} \mathrm{~m}^{2} / \mathrm{s}^{3}\right)$ and moderate temperature stratification $N^{*}$ $=0.35\left(N=0.013 \mathrm{~s}^{-1}\right)$ represents typical environmental conditions at cruise altitude. Stronger and weaker (zero) eddy dissipation rates and temperature stratifications, which are not representative for cruise conditions, are considered around the baseline case. In particular, the cases with strong turbulence $\left(\varepsilon^{*}=0.23\right)$ and strong temperature stratification $\left(N^{*}=1\right)$ would rather be expected in the atmospheric boundary layer than in the tropopause region at cruise altitude.

To generate a turbulence field with a certain value of eddy dissipation rate, an initial velocity field is prescribed based on the stochastic noise generation approach using the von Karman and Pao spectrum. ${ }^{44}$ Then, LES of decaying turbulence is performed in a pre-run until the eddy dissipation rate reaches its maximum value in time. At that time, the energy spectrum has formed a $-5 / 3$ slope of the inertial subrange in neutrally stratified cases $\left(N^{*}=0\right)$. In stably stratified cases $\left(N^{*}>0\right)$, the vertical motion of the flow is suppressed by buoyancy effects and the flow field becomes anisotropic exhibiting horizontally two-dimensional structures (see Fig. 1) (Ref. 45). As can be seen in Fig. 3 for the weakly stably stratified baseline case, the resulting energy spectrum at $t^{*}=90$ appears slightly steeper than the $-5 / 3$ slope.

\section{RESULTS AND DISCUSSION}

\section{A. Temporal evolutions of vortex parameters and topology}

In this section, the temporal developments of vortex circulation and descent distances as well as representative flow and passive tracer fields are presented to provide an overview on the major effects of the different ambient atmospheric conditions listed in Table I on wake vortex evolution.

Figure 4(a) shows the temporal evolution of vortex circulation $\Gamma^{*}$ evaluated in the transverse half of the domain, where $\Gamma^{*}$ is normalized by its initial value $\Gamma_{0}$. In the neutrally stratified case the circulation is conserved until vortex reconnection at $t^{*}=5.6$ (see Fig. 6) and then quickly decreases. At $t^{*}=10$, the vortex ring reconnects once again and transmutes into double rings. The counter-signed axial components of vorticity of one vortex ring in the half domain cancel each other such that the circulation almost vanishes. In the stably stratified cases, the circulation $\Gamma^{*}$ oscillates with the Brunt-Väisälä frequency and even becomes negative. The circulation oscillations result from a superposition of the vorticity of the primary vortex and counter-rotating baroclinic vorticity generated by the baroclinic torque. ${ }^{8,46}$ In the strongly stratified case $\left(\varepsilon^{*}=0.01, N^{*}=1.0\right)$, $\Gamma^{*}$ reaches a minimum at $t^{*}=\pi$ followed by a maximum at $t^{*}=2 \pi$ and another minimum at $t^{*}=3 \pi$. Also the three cases with moderate stratification $\left(N^{*}=0.35\right)$ reach a circulation minimum at $t^{*}=\pi / N^{*} \approx 9$, but the individual curves deviate from each other when the vortices link at slightly different times around $t^{*} \approx 5$. 

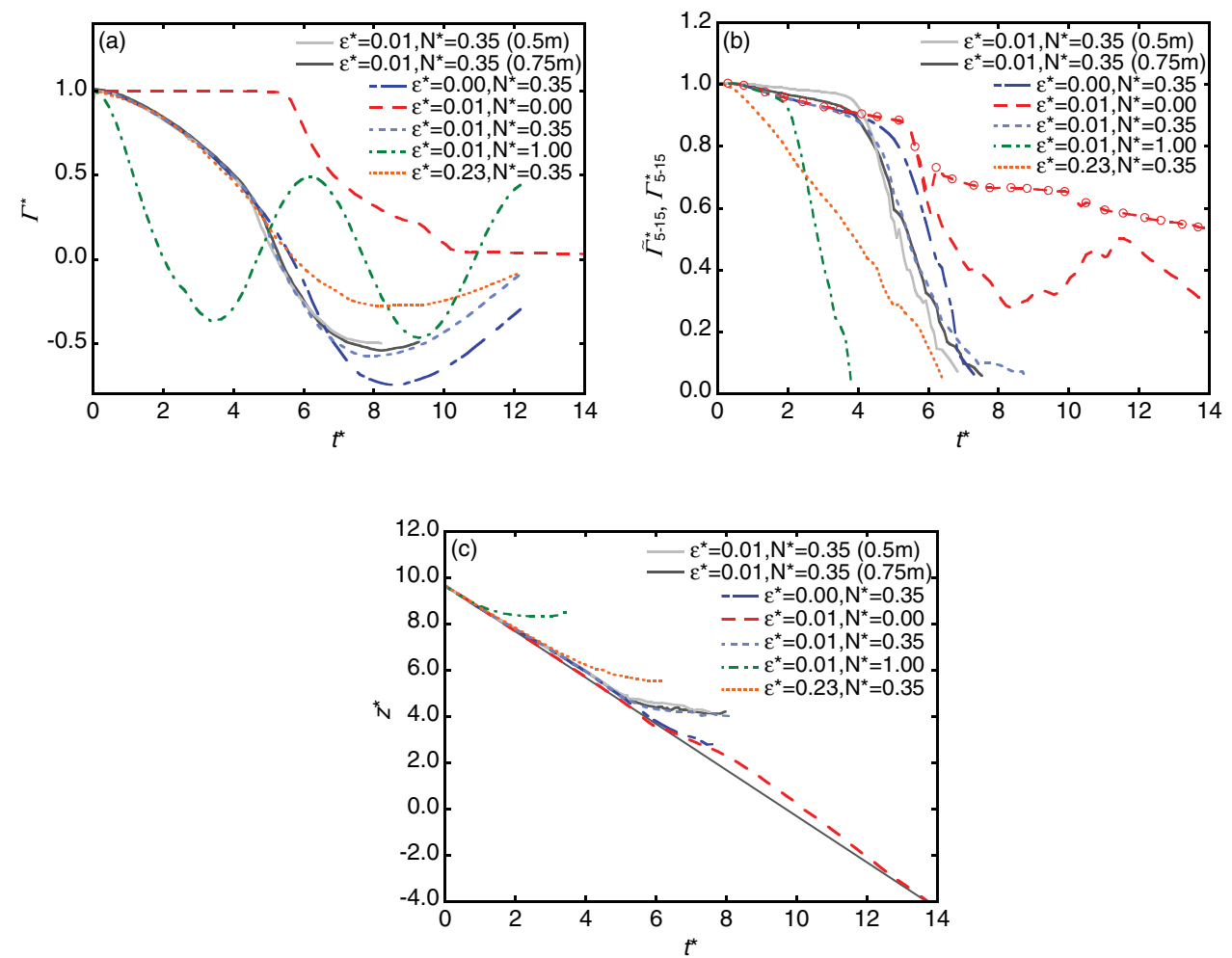

FIG. 4. (Color online) Temporal evolution of (a) longitudinal circulation evaluated over half transverse domain, (b) radiiaveraged circulation along flight direction $\Gamma_{5-15}^{*}$ (lines) and along vortex centerlines $\tilde{\Gamma}_{5-15}^{*}$ (lines with symbols), and (c) vortex descent distances.

In addition to these global circulation characteristics, we consider circulation averaged between vortex radii from 5 to $15 \mathrm{~m}$. The radially averaged circulation has the benefit that it may reveal the evolution of the strength of the primary vortex. Further, $\Gamma_{5-15}^{*}$ allows comparing circulation data from simulation, field measurement, and laboratory experiments. ${ }^{47}$ In Fig. 4(b), the normalized average circulation obtained from the vorticity component in flight direction $\Gamma_{5-15}^{*}$ is denoted by lines, and lines with symbols denote the normalized average circulation determined along the vortex centerline $\tilde{\Gamma}_{5-15}^{*} \cdot \tilde{\Gamma}_{5-15}^{*}$ is estimated based on a predictor-corrector algorithm which realizes robust identification of the vortex centerline. ${ }^{48}$ It thus allows insights into the late evolution of deformed vortex pairs and vortex rings.

The averaged circulations $\Gamma_{5-15}^{*}$ and $\tilde{\Gamma}_{5-15}^{*}$ exhibit two-phase decay characteristics as shown in Fig. 4(b), i.e., the gradual decay in the early diffusion phase is followed by a rapid decay phase, which is due to vortex reconnection and the interaction with secondary vortical structures. ${ }^{16,49}$ The decay rates of the averaged circulation in the diffusion phase are similar and are governed by turbulent diffusion supported by the initially superimposed white noise fluctuations except for the case with $\varepsilon^{*}=0.23$ and $N^{*}=0.35$. In that case, the relatively strong ambient turbulence enhances the decay of circulation from the very beginning. The circulation of the primary vortices is reduced by the work conducted during stretching of baroclinic and turbulent eddies in the environment, ${ }^{16,43}$ and by the turbulent mixing due to the generated secondary vortical structures. In the strongly stratified case $\left(\varepsilon^{*}=0.01, N^{*}=1.0\right)$ the rapid decay starts already at $t^{*}=2$, on the other hand, the moderately stratified cases $\left(\varepsilon^{*}=0, N^{*}=0.35\right)$ and $\left(\varepsilon^{*}=0.01, N^{*}=0.35\right)$ show similar onset times of rapid decay at about $t^{*}=5$. Obviously, strong and even moderate stable stratification have a dominant effect on vortex decay compared to the impact of weak turbulence. The neutrally stratified weakly turbulent case $\left(\varepsilon^{*}=0.01, N^{*}=0\right)$ features a relatively sharp kink at the end of the diffusion phase followed by a recovery of $\tilde{\Gamma}_{5-15}^{*}$ reflecting the reorganization of the vortex after ring formation (see Fig. 6) (Ref. 50). Then, a third phase of gradual decay appears corresponding to a long-lived vortex 
ring whose decay rate of $\tilde{\Gamma}_{5-15}^{*}$ is similar to that in the diffusion phase. ${ }^{48}$ After $t^{*}=7$, the averaged circulation along flight direction $\Gamma_{5-15}^{*}$ exhibits much smaller values than $\tilde{\Gamma}_{5-15}^{*}$, because only small segments of the vortex ring are still parallel to flight direction.

Figure 4(c) illustrates that the vortices initially descend with their theoretical initial descent speed, $w_{0}$, for all environmental conditions despite of the gradual circulation decay. In the neutrally stratified case, the circulation initially decreases mostly on small radii in the 5-15 m range and not on larger radii on the order of the vortex separation $b_{0}$. On the other hand, since vortex separation decreases during vortex descent in stably stratified cases, ${ }^{8,46,51}$ the decrease of the mutually induced velocity and the reduced vortex separation compensate each other leading to constant vortex descent speed in the diffusion phase. After ring formation at $t^{*} \approx 6$ for the case with $\varepsilon^{*}=0.01$ and $N^{*}=0$, the descent speed is slightly reduced but recovers subsequently according to the particular shape of the vortex rings (see Fig. 6). The double rings feature a descent speed of $1.2 w_{0}$ corresponding to the expected descent speed of vortex rings at high Reynolds numbers. ${ }^{14}$

The buoyancy force acting on the descending vortex oval effectively limits vortex descent in the stably stratified cases. For the strong stratification case $\left(N^{*}=1\right)$, the maximum descent amounts to $1.1 b_{0}$ and in the moderately stratified baseline case $\left(\varepsilon^{*}=0.01, N^{*}=0.35\right)$ the descent distance reaches $5.9 b_{0}$. The times when the maximum descent distances are reached correlate well with the times when the respective half-plane circulations in Fig. 4(a) become zero and change sign. The excessive descent distances of more than $16 b_{0}$ (not shown; the simulation was stopped at $16 b_{0}$ although the vortex rings still kept descending) in the neutral atmosphere reaching far beyond the vertical aircraft separation of $1000 \mathrm{ft}$ in reduced vertical separation minimum (RVSM) airspace are prevented by the stable temperature stratification typically prevailing in the tropopause region. ${ }^{52}$

Figures $4(\mathrm{a})-4(\mathrm{c})$ also show results using finer meshes of 0.75 and $0.5 \mathrm{~m}$ for the baseline case $\left(\varepsilon^{*}=0.01, N^{*}=0.35\right)$. Figure 4(b) indicates that the gradual decay of $\tilde{\Gamma}_{5-15}^{*}$ in the diffusion phase is slightly reduced by using the finer meshes. This is because the growth of vortex core radius and the corresponding decrease of tangential velocity are small in these cases. Note that these differences mainly come from white noise added near vortex core. The influence of the mesh resolution on global vortex characteristics such as a time of linking and descending distances is small compared to that of different atmospheric conditions.

The tendencies seen in the evolutions of vortex circulation and descent can be further explained with visualizations of the flow and passive tracer fields. Figure 5 shows iso-surfaces of vorticity magnitude $\left(\left|\omega^{*}\right|=40\right.$, opaque, blue) and tracer concentration ( $c=0.2$, transparent, green) at several instants of time for the baseline case $\left(\varepsilon^{*}=0.01, N^{*}=0.35\right)$. The passive tracer has been initialized in the half-oval cross-section with a maximum value of 1.0 as shown in Fig. 2. In the stably stratified environment baroclinically produced secondary vortices appear along the vortex oval and generate vertical vorticity streaks between the primary vortices. ${ }^{40}$ This enhances the generation of secondary vortices wrapping around the primary vortices. Vortex reconnection at $t^{*}=4.9$ and subsequent vorticity cancellation at the reconnection positions cause the decrease of circulation and the detrainment of passive tracer at the reconnection positions. The vortex ring at $t^{*}=6.1$ is highly disturbed by the secondary vortices resulting in rapid circulation decrease (see Fig. 4).

The role of the baroclinic secondary vortices becomes evident from a comparison of the cases $\left(\varepsilon^{*}=0.01, N^{*}=0.35\right)$ and $\left(\varepsilon^{*}=0.01, N^{*}=0\right)$. In a neutral atmosphere a large part of the averaged circulation may persist after ring formation [see Fig. 4(b)], i.e., the ring formation itself does not decrease the average circulation along the vortex ring $\tilde{\Gamma}_{5-15}^{*}$ except for the loss of circulation due to vortex reconnection. Correspondingly, clearly preserved and smooth vortex ring structures can be seen in Fig. 6. However, the absence of the baroclinic vorticity structures reveals another disturbance provoked by vortex reconnection. Helical vorticity structures ${ }^{21,53}$ propagate from the reconnection points along the vortex cores as in Figs. 6(a)-6(c) and collide midway at $t^{*}=6.4$. Figure 6(f) indicates that the disturbed vortex ring reorganizes subsequently and widens in spanwise direction. At $t^{*}=10$, the elongated vortex ring links another time and two almost circular vortex rings appear. The double rings approach each other and merge again into a single vortex ring at $t^{*}=14.3$. During the entire process, a large part of passive tracer is trapped by the vortex ring. The photograph of a 

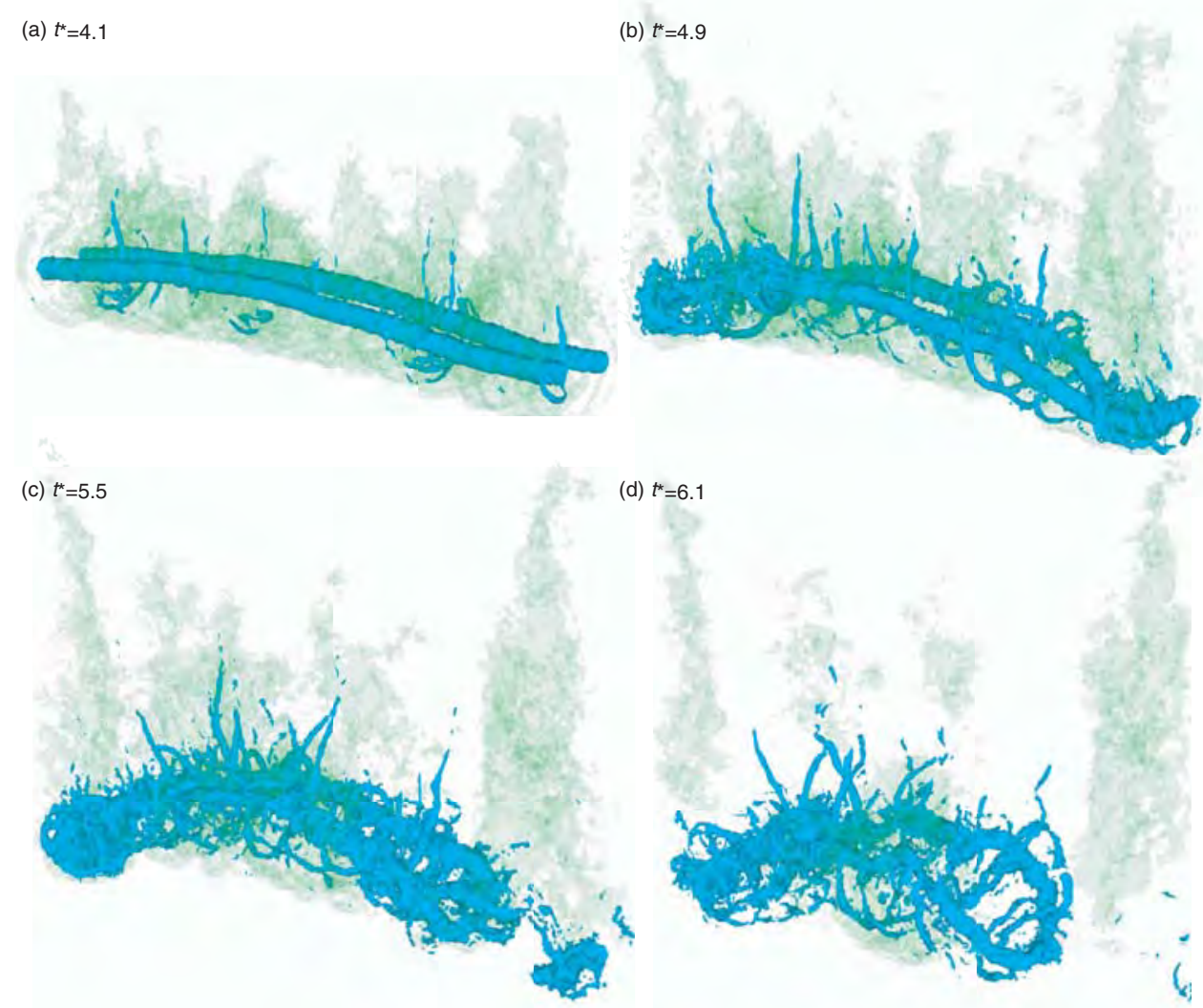

FIG. 5. (Color online) Perspective view of vorticity magnitude (opaque, blue) and passive tracer (transparent, green) iso-surfaces at several vortex ages for the baseline case with $\varepsilon^{*}=0.01$ and $N^{*}=0.35$ (enhanced online). [URL: http://dx.doi.org/10.1063/1.3684990.1] [URL: http://dx.doi.org/10.1063/1.3684990.2]

contrail in Fig. 7 shows the formation of double rings during the early $\infty$-shape phase [see Figs. 6(g) and 7(a)] and of fully developed circular vortex rings [see Figs. 6(h) and Fig. 7(b)]. The formation of double rings where the reconnection plane is rotated by $90^{\circ}$ can be seen in Fig. 7(c). This type of reconnection of vortex rings has been noticed in low Reynolds number experiments. ${ }^{14}$ Further the bone-shaped vortex ring stage in Figs. 6(d) and 6(e) can be observed in Fig. 7(d). Possibly, the photo shows the helical vorticity structure on the left part of the elongated vortex ring [Fig. 7(e)]. Note that the propagating helical vorticity structures are also observed in the case $\left(\varepsilon^{*}=0.01\right.$, $N^{*}=0.35$ ) after vortex reconnection. Since propagating helical vorticity structures locally broaden vortex core radius ${ }^{21}$ they interact with baroclinically produced secondary vortical structures preventing a recovery of vortex structure seen after vortex reconnection in the case $\left(\varepsilon^{*}=0.01\right.$, $\left.N^{*}=0\right)$.

In the case with strong ambient turbulence $\left(\varepsilon^{*}=0.23, N^{*}=0.35\right)$ turbulent eddies are rapidly stretched by the primary vortices and develop into secondary vortices as shown in Fig. 8 that resemble the secondary vortices that were generated from baroclinically produced vorticity at later vortex ages. A vortex ring also appears at $t^{*}=5.2$ although it is already disturbed. As a consequence of the intense mixing caused by the environmental turbulence and the secondary vortices a large part of passive tracer is detrained before vortex reconnection. The vortex evolutions of the cases $\left(\varepsilon^{*}=0, N^{*}=0.35\right)$ and $\left(\varepsilon^{*}=0.01, N^{*}=0.35\right)$ appear similar because the moderate stable stratification dominates the weak ambient turbulence. For $\varepsilon^{*}=0.01$ and $N^{*}=1.0$, strong stratification rapidly induces strong baroclinic vortices. Since the vortices approach each other during their descent, ${ }^{46,51}$ the secondary vortices appear with shorter periods than in the weakly stratified cases and the rapid decay is initiated before vortex reconnection. 


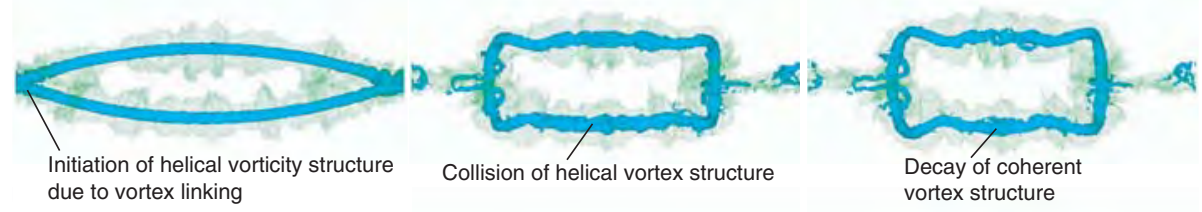

(d) $t^{*}=7.0$

(e) $t^{\star}=7.3$

(f) $t^{*}=8.2$
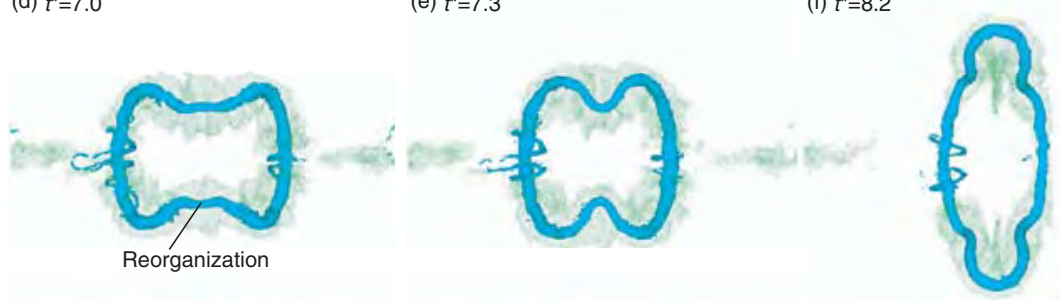

(g) $t^{*}=10.0$
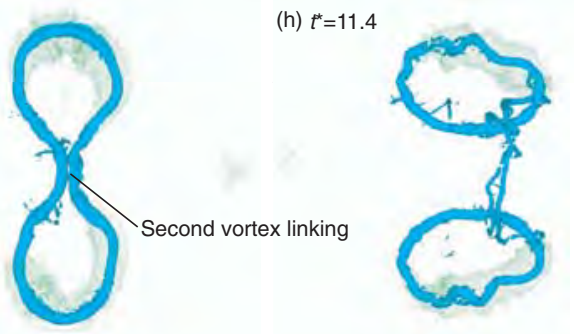

(i) $t^{\star}=14$.

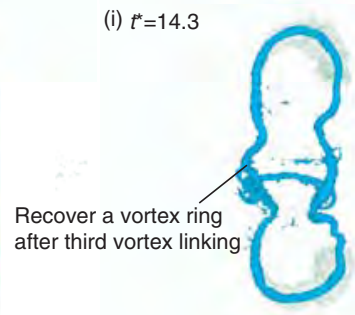

FIG. 6. (Color online) Top view of vorticity magnitude (opaque, blue) and passive tracer (transparent, green) iso-surfaces at several vortex ages for $\varepsilon^{*}=0.01$ and $N^{*}=0$ (enhanced online). [URL: http://dx.doi.org/10.1063/1.3684990.3] [URL: http://dx.doi.org/10.1063/1.3684990.4]

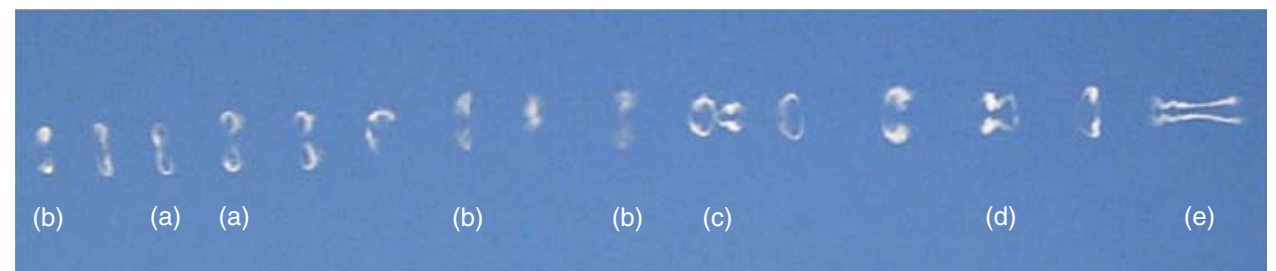

FIG. 7. (Color online) Photograph of a contrail featuring double vortex rings: flight direction from left to right. Photo taken from ground on November 16, 2006 at 8:53 by S. Lüke.

(a) $t^{*}=2.3$

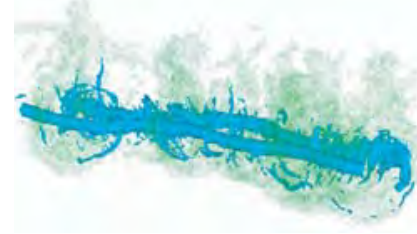

(b) $t^{\star}=4.1$

(c) $t^{*}=5.2$

FIG. 8. (Color online) Perspective view of vorticity magnitude (opaque, blue) and passive tracer (transparent, green) isosurfaces at several vortex ages for $\varepsilon^{*}=0.23$ and $N^{*}=0.35$. 


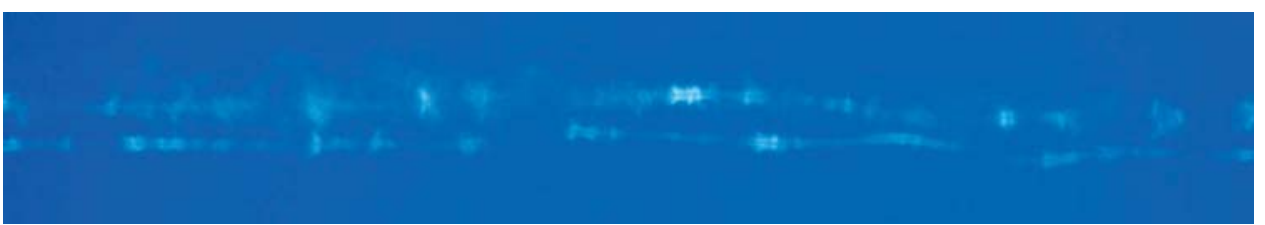

FIG. 9. (Color online) Photograph of a contrail generated by a two-engine commercial jet airplane indicating the vortex bursting phenomenon. Photo taken from ground on September 30, 2007 at 17:13 by F. Holzäpfel. Reprinted with permission from F. Holzäpfel, T. Misaka, and I. Hennemann, "Wake-vortex topology, circulation, and turbulent exchange processes," in Proceedings of the 2nd AIAA Atmospheric and Space Environments Conference, 2-5 August, Toronto, Ontario (AIAA, 2010), Paper No. 2010-7992. Copyright (C 2010, American Institute of Aeronautics and Astronautics, Inc.

\section{B. Secondary vortical structures, vortex bursting, and vortex funnels}

In this section, we discuss the vortex busting phenomenon that can occasionally be observed when wake vortices are visualized by smoke or contrails. ${ }^{17-20,22}$ Vortex bursting is referred to as a phenomenon where the region marked by ice crystals or smoke contracts in portions along the vortices and expands in others ${ }^{22}$ (see Fig. 9). It has been argued that vortex bursting is not related to vortex decay because the vortex pair keeps descending after the occurrence of vortex bursting. In addition, the characteristic tracer distribution corresponding to vortex bursting appears independent from vortex reconnection, i.e., it is observed before vortex reconnection. Moet et al. ${ }^{21}$ associate vortex bursting with an abrupt change of flow structure in the vortex core that is observed when traveling helical vorticity structures collide (see Fig. 6). The traveling helical vorticity structures are connected with pressure waves and are initiated by vortex reconnection; therefore, the collision and subsequent local vortex decay are observed after vortex reconnection. To the authors' knowledge, simulations of vortex bursting including a visualization of the flow by a passive tracer have not been conducted so far, therefore, the relation between the flow structures of vortex bursting and their visual appearance remains open. In this section, we employ flow and passive tracer fields in order to discuss the relation between vortex bursting visualized by passive tracers and the underlying flow structures.

Figure 10(a) shows vorticity magnitude $\left(\left|\omega^{*}\right|=32\right.$, opaque, blue) and passive tracer iso-surfaces ( $c=0.25$, transparent, red and green) for the baseline case with $\varepsilon^{*}=0.01$ and $N^{*}=0.35$ at $t^{*}$ $=4.6$. Distinct tracer fields have been superimposed to the upper and lower vortex in their respective half-oval cross-sections that are visualized with different colors. In Fig. 10, an apparent redistribution of passive tracer along the vortex tubes can be observed. Similar as in the photograph in Fig. 9, the passive tracer is preserved around the vortex cores but has been detrained on larger radii along extended sections of the vortex tubes. Figure 10(a) indicates that the tracer is detrained along the vortex tubes where secondary vortices are formed. At some locations the tracer apparently accumulates featuring expanded structures that are commonly associated with vortex bursting. Since the redistribution occurs before vortex reconnection, it cannot be caused by traveling helical vorticity

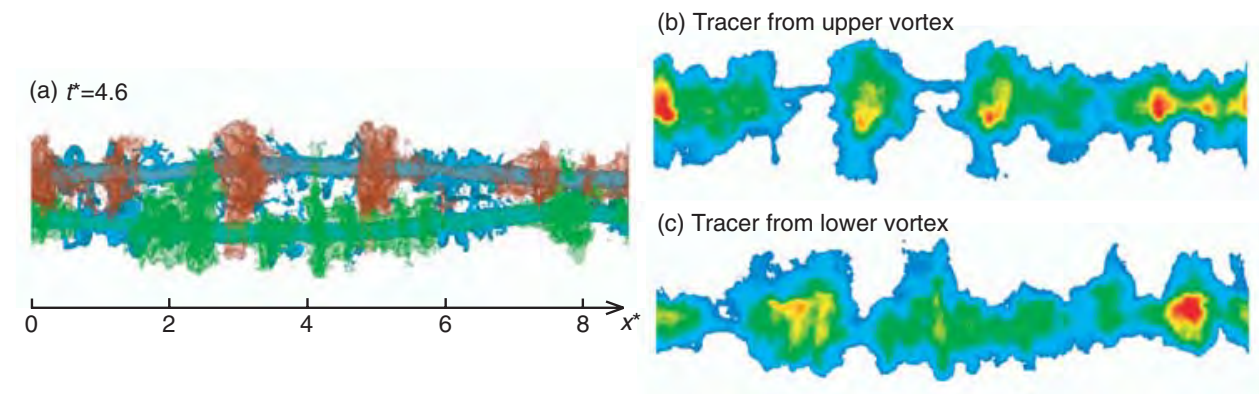

FIG. 10. (Color online) Top view of iso-surfaces of (a) vorticity magnitude (opaque, blue) and passive tracers (transparent, red and green) and projection plots of passive tracers of (b) upper vortex and (c) lower vortex at $t^{*}=4.6$ for the baseline case with $\varepsilon^{*}=0.01$ and $N^{*}=0.35$. 


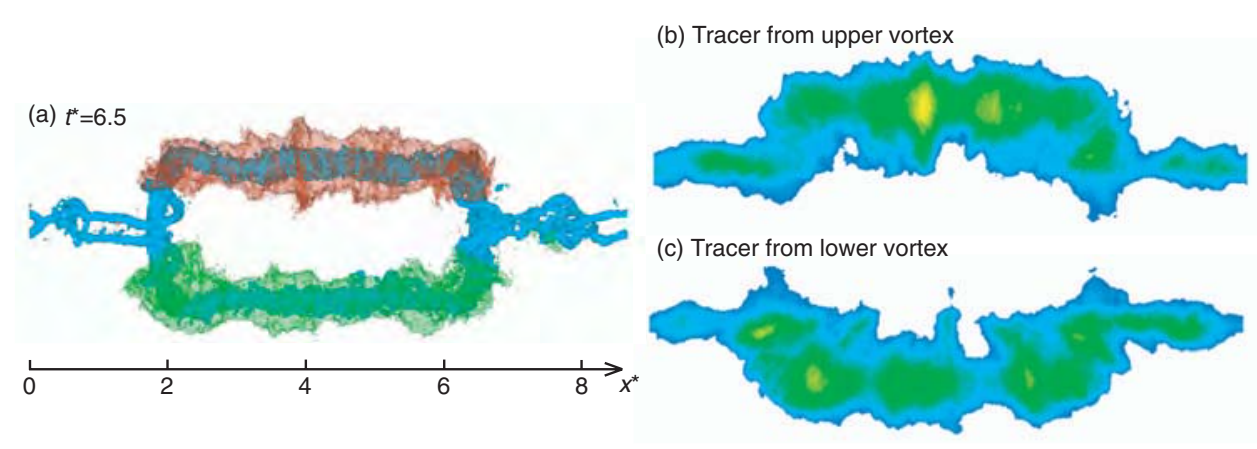

FIG. 11. (Color online) Top view of iso-surfaces of (a) vorticity magnitude (opaque, blue) and passive tracers (transparent, red and green) and projection plots of passive tracers of (b) upper vortex and (c) lower vortex at $t^{*}=6.5$ for $\varepsilon^{*}=0.01$ and $N^{*}=0$.

structures initiated by vortex reconnection. Figures 10(b) and 10(c) show projection plots generated by the integration of passive tracer in vertical direction where the color coding of the plot is arbitrarily set. Compared to the iso-surfaces shown in Fig. 10(a), the projection plots in Figs. 10(b) and 10(c) should be closer to a view of real wake vortices visualized by smoke or contrails. Albeit somewhat less distinct, the projection plots also indicate that a certain amount of passive tracer is trapped within the vortex cores while it is expanded at other positions.

Figures 11(a)-11(c) show the corresponding plots after vortex reconnection for the neutrally stratified case $\left(\varepsilon^{*}=0.01, N^{*}=0\right)$ applying the same levels and color codings as in Fig. 10. At $t^{*}=6.5$, the propagating helical vorticity structures collide, however, the influence of the transient loss of coherent vortex flow on the distribution of passive tracer is weak. The collision of helical vorticity structures leads to a slightly increased tracer concentration only in the middle of the upper vortex. Note that the passive tracer is effectively detrained in the areas where the vortices have linked. Figures 12(a)-12(c) show the corresponding plots for the case with stronger turbulence and moderate stratification $\left(\varepsilon^{*}=0.23, N^{*}=0.35\right)$. Here secondary vortices are quickly generated by the stretching of the turbulent eddies and the baroclinic vorticity structures as seen in Fig. 12(a). As a result, large and distinct vortex bursting structures appear already at $t^{*}=2.2$ that resemble the pancake shape mentioned by Spalart. ${ }^{22}$ Also the projection plots in Figs. 12(b) and 12(c) illustrate the pronounced distribution of passive tracer occurring before vortex reconnection as in the baseline case.

Figures 13(a)-13(d) show quantities evaluated in ring segments extending within radii from 15 to $25 \mathrm{~m}$ around the vortex centerline (see Fig. 14 for definition of the control-volume) of the upper vortex depicted in Figs. 10(a). These different quantities are plotted along the identified positions of the descending vortex centerline. Secondary vortical structure pairings can be identified by tangential

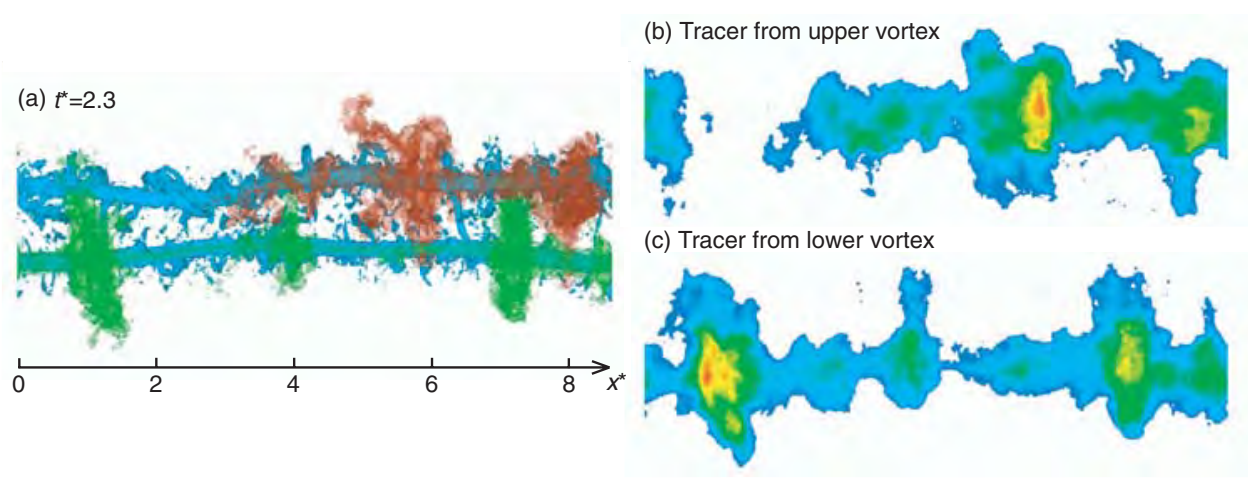

FIG. 12. (Color online) Top view of iso-surfaces of (a) vorticity magnitude (opaque, blue) and passive tracers (transparent, red and green) and projection plots of passive tracers of (b) upper vortex and (c) lower vortex at $t^{*}=2.3$ for $\varepsilon^{*}=0.23$ and $N^{*}=0.35$. 

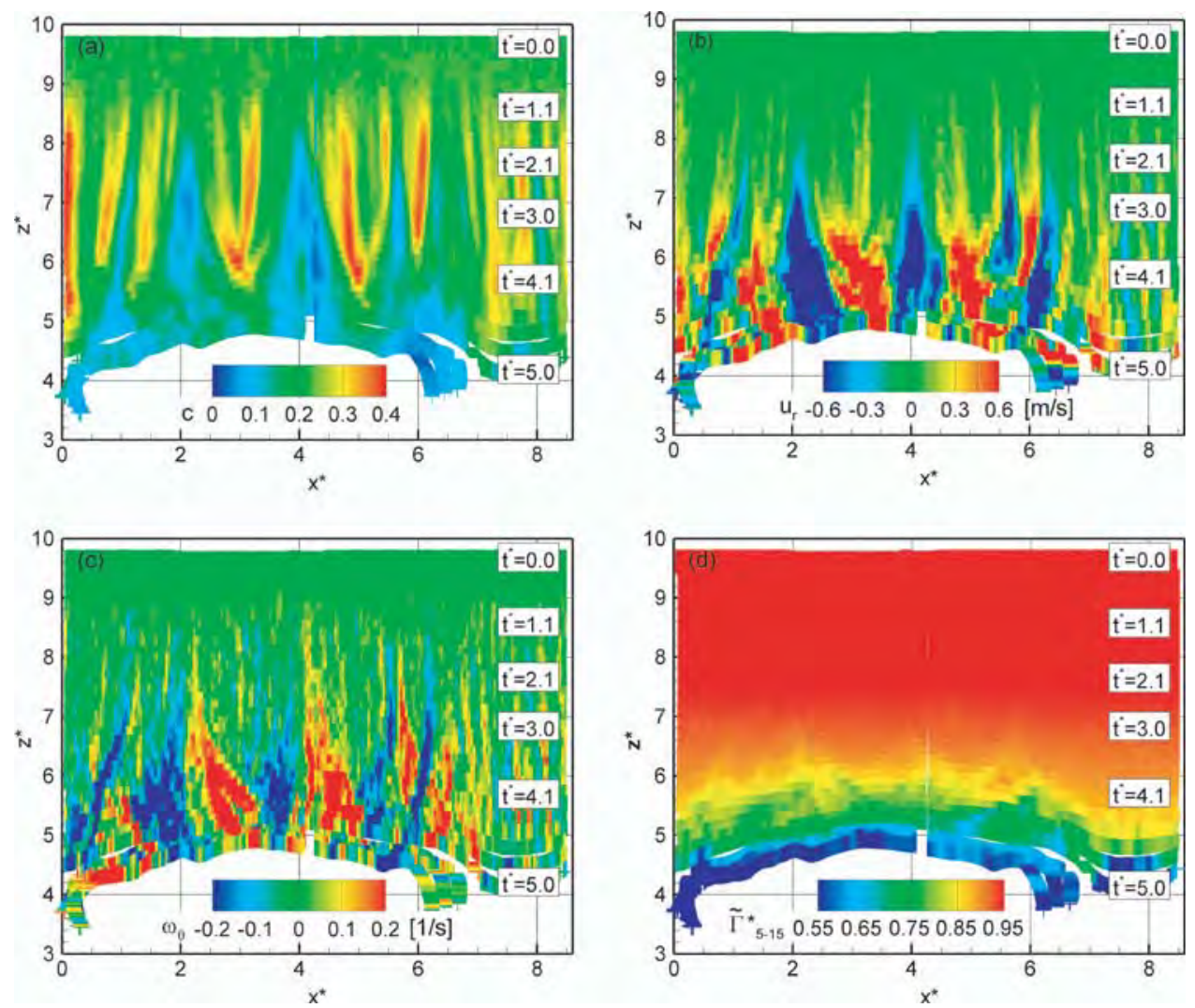

FIG. 13. (Color online) Distributions of (a) passive tracer, (b) radial velocity, and (c) tangential vorticity averaged between radii from 15 to $25 \mathrm{~m}$ and (d) circulation $\tilde{\Gamma}_{5-15}^{*}$ plotted along the identified positions of the descending vortex centerline for $\varepsilon^{*}=0.01$ and $N^{*}=0.35$. Corresponding vortex ages are included for convenience.

vorticity in Fig. 13(c) that approach each other while they are gaining strength. For example, one secondary vortical structure originates from $\left(x^{*} \approx 2.2, z^{*} \approx 8\right)$ traveling toward to the right while its counter-rotating mate is generated at $\left(x^{*} \approx 3.8, z^{*} \approx 7.5\right)$ traveling toward to the left [see Fig. 13(c)]. The secondary vortical structure collides at $\left(x^{*} \approx 3.3, z^{*} \approx 5.5\right)$. Figure 13(a) illustrates that the traveling secondary vortical structures proceed along with an accumulation and thinning of the passive tracer. $\vee$-shaped tracer accumulations appear in front of the secondary vortical structures and $\wedge$-shaped tracer thinning exist behind them.

The radial velocities displayed in Fig. 13(b) indicate that high concentrations in the evaluated ring segments are connected to positive radial velocities in front of the secondary vortical structure, i.e., fluid with high tracer concentrations is transported from the vortex cores to larger radii. Conversely, negative radial velocities transport fluid with low concentrations from the ambient flow to small radii. And the collision of two counter-rotating secondary vortical structure causes the pancake-shaped structures associated with vortex bursting. Compare, for example, the concentration maxima at $\left(x^{*}\right.$ $\left.=3, z^{*}=6\right)$ and $\left(x^{*}=5, z^{*}=6\right)$ in Fig. 13(a) and the corresponding tracer structures in Fig. 10(a). The $\tilde{\Gamma}_{5-15}^{*}$ evolution in Fig. 13(d) clearly indicates that the process causing the tracer redistribution does not influence the local vortex strength. Although the visual appearance of vortex bursting may suggest local vortex weakening, it is not associated with local vortex decay or the vortex breakdown phenomenon.

The effect of the secondary vortical structure on the redistribution of passive tracer is explained in more detail in Fig. 15. Figure 15(a) depicts a perspective view on the wake vortex pair and a visualization of its flow field in terms of streamlines in the background. The right primary vortex is surrounded by a vortex ring which induces itself a propagation velocity from right to left. This vortex ring represents spiral-shaped azimuthal vorticity structures that are generated from 


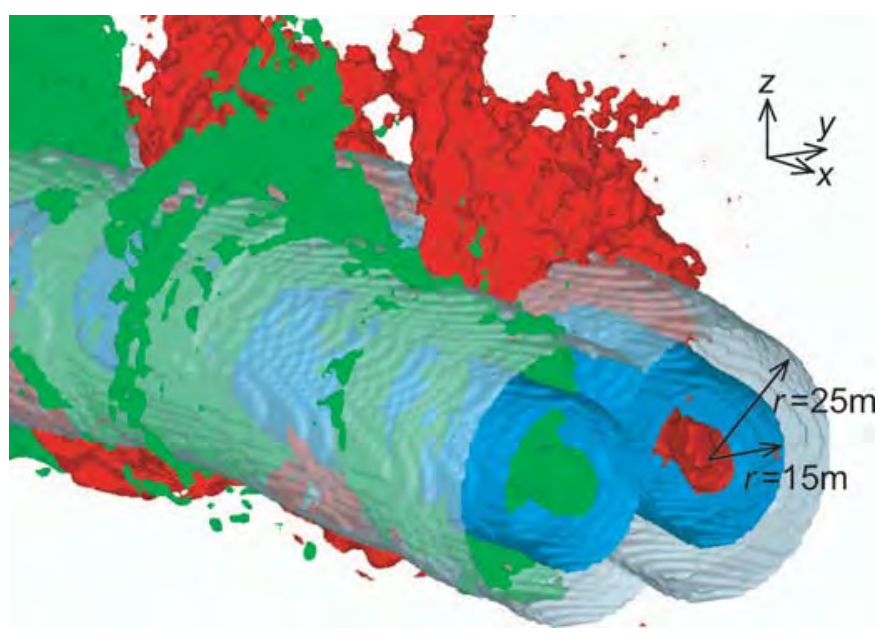

FIG. 14. (Color online) Distribution of passive tracers (opaque, red and green) and a tubelike control volume defined between radii of 15 and $25 \mathrm{~m}$ shown by opaque (blue) and transparent (light blue) surfaces, respectively, where the quantities in Figs. 13(a)-13(c) are evaluated.

environmental turbulence and baroclinic vorticity in a stably stratified environment by vortex stretching mechanisms. ${ }^{16,40}$ Figure 15 (b) sketches a cross section through the right primary vortex including a streamline plot of the vortex ring. When coherent azimuthal secondary vortical structures (represented by the vortex ring) are generated, they induce themselves a propagation velocity along the primary vortices. During this propagation, they transport ahead passive tracer from the vortex center (high tracer concentration) toward larger radii as illustrated by the streamline pattern of the vortex ring in Figs. 15(a) and 15(b). In this way, the tracer reaches the boundaries of the primary vortex oval where it can be detrained. Therefore, the concentration ahead of the propagating secondary vortical structures is increased [ $\vee$-shaped structures in Fig. 13(a)] and it is decreased behind them [^-shaped structures in Fig. 13(a)]. When two secondary vortical structures collide, the radial tracer transport is increased temporarily resulting in the generation of the pancake-shaped structures. The secondary vortical structures also enable transport of swirling flow toward larger radii (and in turn fluid with lower tangential velocities toward smaller radii) intensifying systematically the turbulent diffusion of the primary vortices from the interior to the exterior flow.

The conceptual description of these processes is confirmed by observations: Brown ${ }^{19}$ observed in his flight experiments funnel-shaped ice crystal features along the vortex cores that were frequently surrounded by the pancake-shaped structures. Figure 16 shows a photo of such funnel-shaped features without the surrounding pancake-shaped structures. Figure 17 illustrates a close-up view of Fig. 10 where several levels of tracer iso-surfaces are visualized instead of vorticity magnitude to see
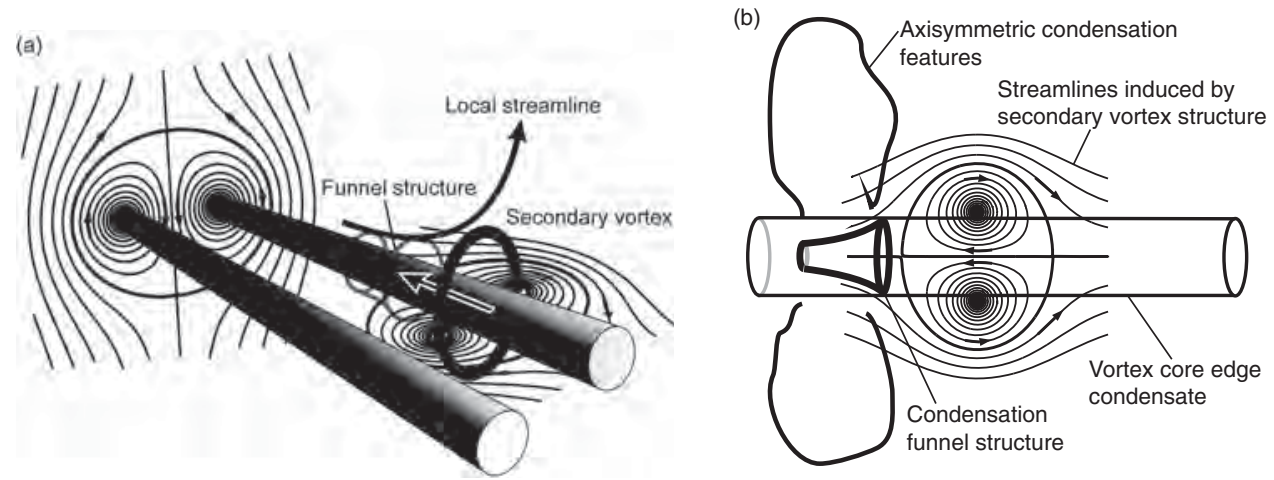

FIG. 15. Schematic of (a) local passive tracer transport by secondary vortices, (b) cross-sectional sketch inspired by Brown. ${ }^{19}$ 


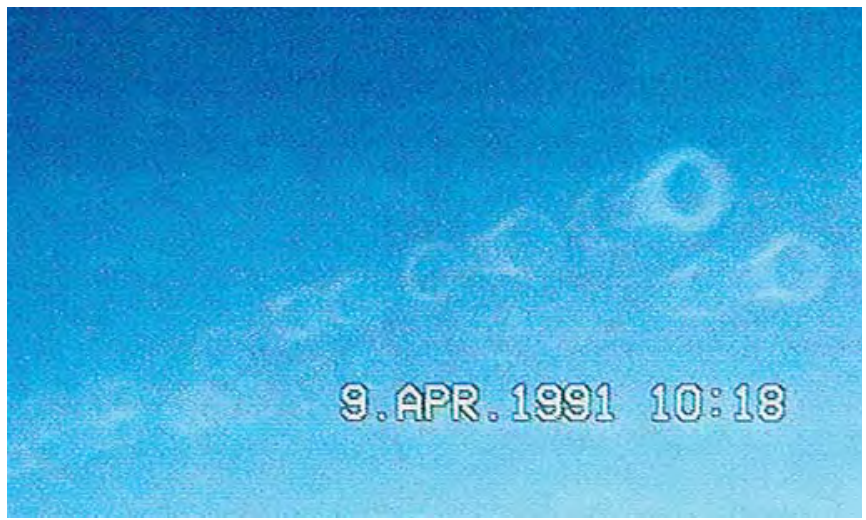

FIG. 16. (Color online) Photograph of funnel-shaped ice crystal features generated by a McDonnell Douglas DC-10 aircraft taken from DLR Falcon research aircraft.

the tracer distribution inside of the pancake-shaped structures. The pancake-shaped structures are represented by low passive tracer concentrations visualized in transparent surfaces (red and green). Higher tracer concentrations (opaque, red and green) reveal the outer surface of funnel-shaped structures surrounded by the pancake-shaped structures. The relative positions between the funnel features and the pancake features are similar to Brown's sketch in Fig. 15(b). The shape of these funnels matches the streamline pattern ahead of a secondary vortex ring, i.e., the diverging streamlines ahead of the propagating secondary vortical structure may widen the ice crystal distribution around the vortex cores.

In Fig. 18, the tracer distributions associated with vortex bursting at $t^{*}=4.6$ for the baseline case are compared in terms of tracer initialization and mesh resolution. Figures 18(a), 18(b), and 10(b) show projection plots of tracers initialized within vortex core, full-oval, and half-oval cross-sections, respectively. The tracer redistribution due to secondary vortical structures is clearly seen for all the different tracer initializations. On the other hand, Figs. 18(a), 18(c), and 18(d) show the influence of the mesh resolution on the tracer distribution. Here, the vortex core initialization is considered because the evolution of the tracer confined in the tight vortex cores is affected by the difference of the mesh resolution compared to the other initializations. A larger amount of tracer is kept within the vortex core when a finer mesh is used; however, the tracer redistribution along the vortex tube is seen in all cases. The tracer redistribution caused by secondary vortical structures takes place robustly

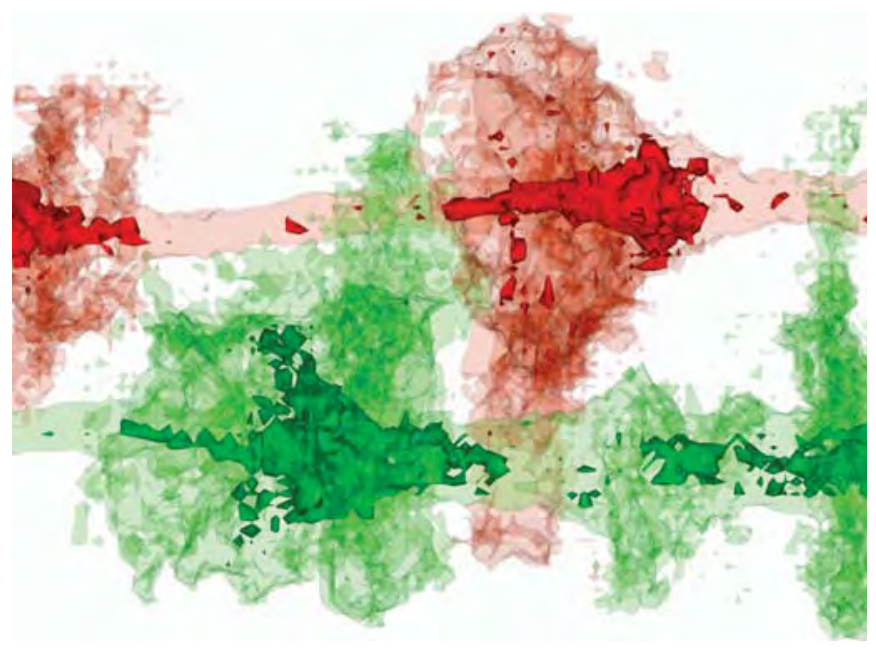

FIG. 17. (Color online) Iso-surfaces of passive tracers (upper opaque, red: $c=0.43$, upper transparent, red: $c=0.25$, lower opaque, green: $c=0.5$, lower transparent, green: $c=0.25)$ at $t^{*}=4.6$ for the baseline case with $\varepsilon^{*}=0.01$ and $N^{*}=0.35$. 
(a) Vortex core initialization (1.0m mesh)

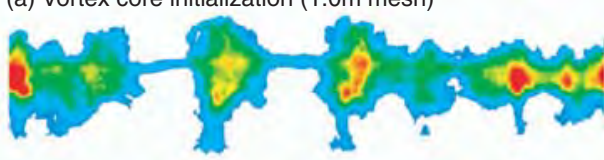

(c) Vortex core initialization $(0.5 \mathrm{~m}$ mesh)

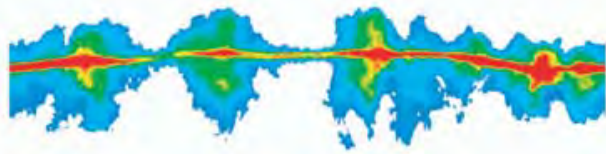

(b) Full-oval initialization (1.0m mesh)

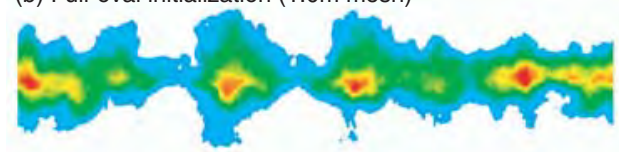

(d) Vortex core initialization $(0.75 \mathrm{~m}$ mesh)

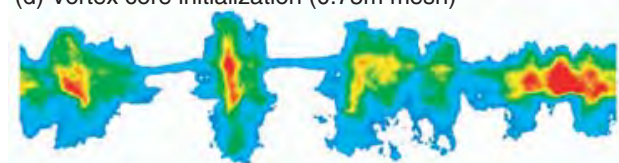

FIG. 18. (Color online) Projection plots of passive tracers of the upper vortex corresponds to Fig. 10(b) with (a) vortex core and (b) full-oval initializations, as well as (c) vortex core initialization of $0.5 \mathrm{~m}$ mesh and (d) that of $0.75 \mathrm{~m}$ mesh at $t^{*}=4.6$ for the baseline case with $\varepsilon^{*}=0.01$ and $N^{*}=0.35$.

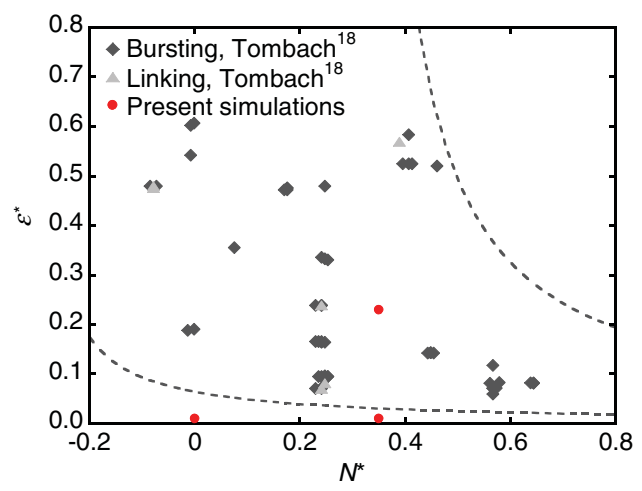

FIG. 19. (Color online) Atmospheric conditions reproduced from Tombach ${ }^{18}$ and the conditions considered in this study

within the ranges of the atmospheric conditions, the tracer initializations, and the mesh resolutions considered here.

Figure 19 shows plots of atmospheric conditions in terms of $\varepsilon^{*}$ and $N^{*}$ reproduced from Tombach $^{18}$ including the conditions of the present simulations. The lines in the figure represent the range of measurement data. ${ }^{18}$ The case $\left(\varepsilon^{*}=0.23, N^{*}=0.35\right)$ where the pancake-shaped tracer structures appear most clearly locates in the center of Tombach's observation conditions. This may indicate that vortex bursting observed in Tombach's experiments is mainly caused by secondary vortical structures generated from ambient turbulence, which coincides with the fact that the flight experiments were conducted near the ground. In Tombach's flight experiments, no relevant dependency of the phenomenon, i.e., vortex linking or bursting, on atmospheric conditions was observed.

Based on the results shown above, the tracer redistribution along the vortices is related to secondary vortical structures produced by baroclinic vorticity and/or strong ambient turbulence which are propagating along the vortex tubes. These secondary vortical structures transport highly concentrated tracer from the vortex centers to large radii where it can be detrained. Pancake-shaped structures appear when counter-rotating secondary vortical structures collide. The secondary vortical structures may also generate funnel-shaped features that have been observed within the pancakeshaped structures. Vortex circulation and vortex core structure are only gradually affected by these processes. On the other hand, the effect of the helical vorticity structures on the tracer distribution is weak compared to the effects of the colliding secondary vortical structures because the former effect is focused on the vortex core area whereas the latter effect extends from the core area to much larger vortex radii.

\section{Tracer detrainment and extent}

As shown in Figs. 5, 6, and 8, the passive tracer trapped by the descending vortex pair or vortex ring is detrained by several phenomena such as secondary vortical structures, vortex reconnection, or 


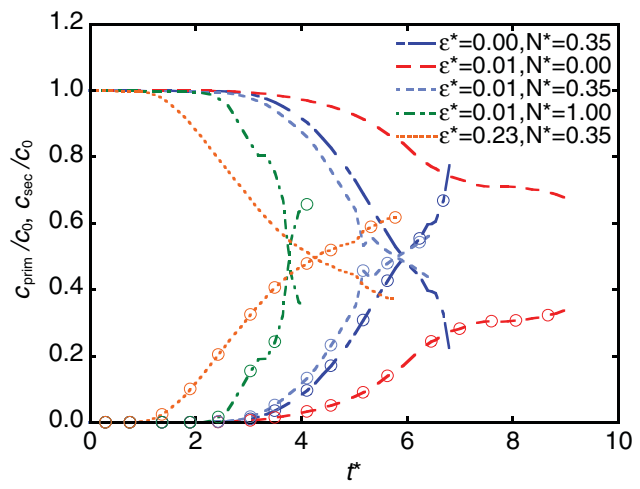

FIG. 20. (Color online) Temporal evolution of normalized passive tracer concentrations in primary (lines) and secondary wakes (lines with symbols).

vortex decay. Previous two-dimensional simulation studies have well explained the basic mechanisms of detrainment in stably stratified environments, where the baroclinic vorticity produced during vortex descent in a stably stratified environment plays a primary role. ${ }^{46}$ The detrained fluid forms a secondary wake which is vertically spreading due to primary vortex descent and buoyancy effects. ${ }^{6}$

Figure 20 illustrates the detrainment of the passive tracer from the primary wake and the corresponding tracer concentration increase in the secondary wake. The figure shows results of the half-oval initialization. A tracer is attributed to the primary wake $c_{\text {prim }}$ when it resides within a cylindrical volume with a radius of $b_{0}$ centered on the identified vortex centerlines. The tracer outside of this control volume is attributed to the secondary wake $c_{\mathrm{sec}}$. This method allows to distinguish between primary and secondary wake for extensively deformed vortices and a vortex ring (see Fig. 21). Figure 20 confirms that the degree of stable stratification dominantly controls the detrainment rate whereas the strength of ambient turbulence mainly impacts the onset time of detrainment. In the case $\left(\varepsilon^{*}=0.01, N^{*}=0\right)$, the passive tracer concentration decreases due to vortex reconnection and the smaller detrainment rate is recovered after this. The vortex detrainment characteristics are well correlated with the decay characteristics of $\tilde{\Gamma}_{5-15}^{*}$ shown in Fig. 4(b). Also, the sequence of the different cases with respect to the onset of rapid circulation decay and the onset of detrainment is the same. The times when the circulation has reached $50 \%$ of its initial value approximately coincide with the times when $50 \%$ of the tracer has been detrained as shown in Fig. 20. In the neutrally stratified case, a large amount of passive tracer is being trapped by the vortex ring during its descent such that the tracer concentration in the primary wake remains above $60 \%$.

The correlation of the tracer detrainment and vortex decay is further substantiated in Fig. 22 which depicts the half-value times of $\tilde{\Gamma}_{5-15}^{*}$ versus the half-value times of passive tracer

(a) top view

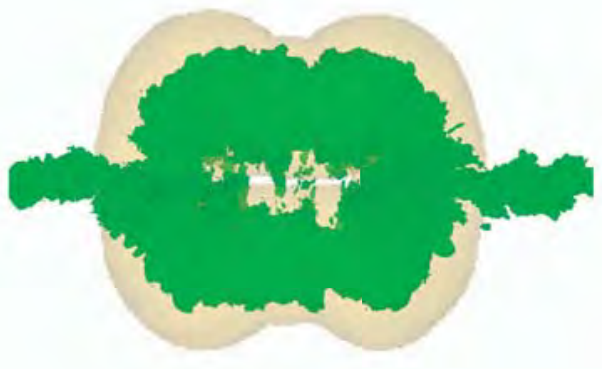

(b) side view

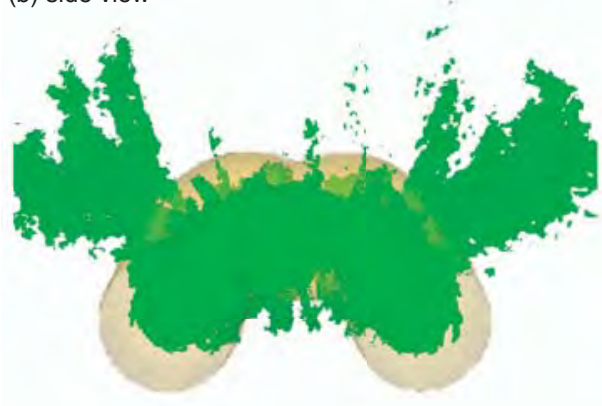

FIG. 21. (Color online) Passive tracer distribution (opaque, green) for the case $\varepsilon^{*}=0.01$ and $N^{*}=0$ at $t^{*}=7.0$ with a control volume (transparent, orange) separating primary and secondary wakes: (a) top view, (b) side view. 


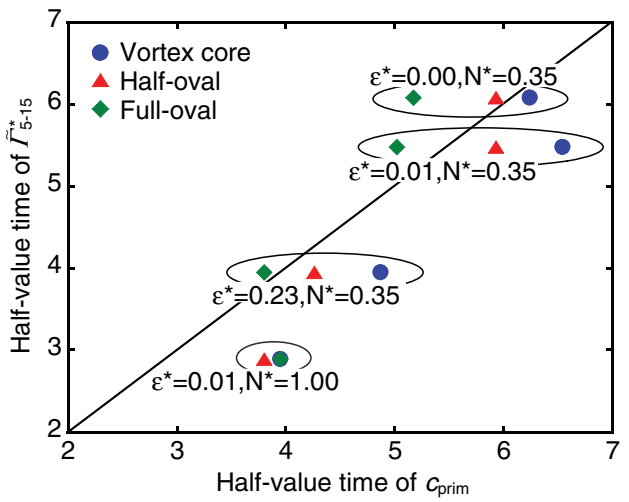

FIG. 22. (Color online) Half-value time of the averaged circulation $\tilde{\Gamma}_{5-15}^{*}$ versus that of the passive tracer concentration in the primary wake $c_{\text {prim }}$ for the three different tracer initializations.

concentrations in the primary wake for the three different tracer initializations. For the initialization in the full-oval, the detrainment starts almost immediately whereas it is delayed for the half oval and slightly further delayed for the tracer initialization within the vortex cores. For the most stably stratified case, the detrainment progresses about one time unit $t_{0}$ slower than the decay because the stagnation of vortex descent [see Fig. 4(c)] interferes with the detrainment mechanism. The tracer initializations within the full oval and the vortex core can be considered as extreme limits embracing realistic scenarios. As a consequence, the similar characteristics of the detrainment rates of the three different initializations indicate a relatively low sensitivity of detrainment rates on the initial tracer distributions within the wake.

Figure 23 shows the temporal evolutions of vertical and horizontal extents of passive tracer concentrations above $5 \%$ of its initial value, where the extents are averaged in flight direction. Wide vertical tracer extents of more than $6 b_{0}$ are found for the cases with no or weak ambient turbulence and moderate stratification $\left(\varepsilon^{*}=0, N^{*}=0.35\right.$ and $\left.\varepsilon^{*}=0.01, N^{*}=0.35\right)$. Wide vertical extents are typically connected to low horizontal extents of the tracer and vice versa. In the neutrally stratified case, the vortex descent distance is larger than in the other cases; however, the vertical spreading of the tracer is relatively small because a large part of the tracer is being trapped by the descending vortex ring and the amount of detrainment is small. In the present five cases, a dominant effect of temperature stratification on the detrainment characteristics is observed. Moderately stable stratification supports the tracer detrainment and the rebound of the detrained fluid such that the tracer is spread along significant vertical dimensions. However, strong stratification inhibits wake vortex descent and vertical spreading of the tracer, instead the horizontal spreading may achieve large values of over $7 b_{0}$.
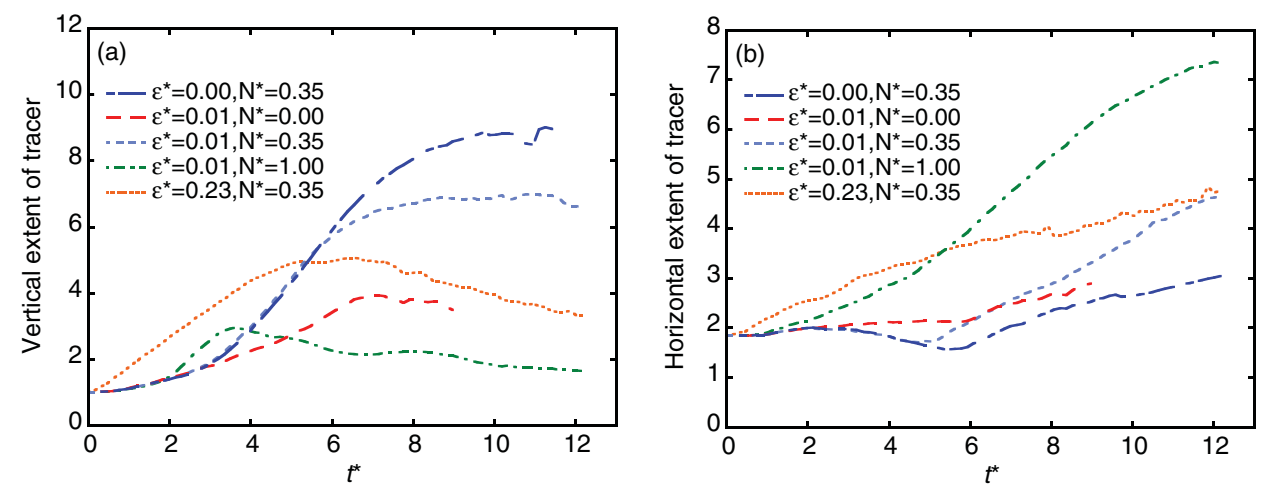

FIG. 23. (Color online) Temporal evolution of (a) vertical and (b) horizontal extents of passive tracer concentrations above $5 \%$ of $c_{0}$ where the extents are averaged in flight direction. The tracer is initialized within the vortex half-oval. 


\section{CONCLUSION}

Large-eddy simulations of a coherent counter-rotating vortex pair in various stably stratified turbulent environments have been conducted and analyzed. Turbulent exchange processes between the vortices and their environment have been investigated employing passive tracers.

In a neutrally stratified and weakly turbulent atmosphere, a long-lived vortex ring forms. The vortex ring broadens and links once again while a short time later the established double rings merge into single vortex ring. These transformations do not prevent the vortex rings to reach excessive descent distances of more than $2500 \mathrm{ft}$ which is far beyond the vertical aircraft separation of $1000 \mathrm{ft}$ prescribed in RVSM airspace. Moderately stable temperature stratification as it is typically prevailing at cruise altitudes is sufficient to significantly reduce vortex lifetimes and descent distances where baroclinic secondary vortical structures lead to rapid vortex decay. In relatively strong environmental turbulence, secondary vortical structures are quickly generated by stretching of turbulent eddies resulting in immediate gradual vortex decay.

Pronounced redistribution processes of passive tracer along the vortex tubes are observed which represents well the vortex bursting phenomenon reported from laboratory and flight experiments. It is suggested that the vortex bursting phenomenon is related to secondary vortical structures produced from baroclinic vorticity and/or ambient turbulence. The secondary vortical structures induce themselves a velocity such that they are propagating along the vortex tubes. At the same time the secondary vortical structures transport passive tracer from the vortex centers to large radii where it can be detrained. Pancake-shaped structures appear when counter-rotating secondary vortical structures approach each other and collide. The secondary vortical structures may also generate funnel-shaped features that have been observed within the pancake-shaped structures or as a solitary phenomenon around the vortex cores. So both effects, vortex bursting visualized by pancake-shaped structures and funnel-shaped vortex core features, are related to the fluid transport caused by propagating secondary vortical structures. On the other hand, vortex circulation is almost not affected by these processes. Pressure waves are triggered by vortex linking and become manifest in helical instabilities in weakly turbulent environments. Collisions of these propagating helical vorticity structures locally impact the coherence of the vortex and reduce its circulation; however, they have only little impact on the tracer distribution. Summary of flow features are listed in Table II.

Detrainment characteristics of a passive tracer have been investigated by considering a control volume along vortex centerlines. The degree of stable stratification dominantly controls the detrainment rate whereas the strength of ambient turbulence mainly impacts the onset time of detrainment. In the neutrally stratified case, a large amount of passive tracer is trapped by the vortex ring during its descent such that the tracer concentration in the primary wake remains above $60 \%$. The tracer detrainment characteristics are well correlated with the vortex decay characteristics. For example,

TABLE II. Summary of flow features observed in the present simulations.

\begin{tabular}{|c|c|c|c|}
\hline & $N^{*}=0.0$ & 0.35 & 1.0 \\
\hline & & - Intermediate vortex lifetime & \\
\hline \multirow[t]{3}{*}{$\varepsilon^{*}=0.0$} & - & Baroclinic SVSs & - \\
\hline & & . Vortex bursting and funnels & \\
\hline & - Long-lived vortex ring & - Intermediate vortex lifetime & - Short vortex lifetime \\
\hline \multirow[t]{4}{*}{0.01} & - Double ring & - Baroclinic SVSs & · No linking \\
\hline & - Excessive vortex descent & . Vortex bursting and funnels & - Intense baroclinic SVSs \\
\hline & - Propagating HVSs & - Propagating HVSs & \\
\hline & & - Immediate circulation decay & \\
\hline \multirow[t]{2}{*}{0.23} & - & - Turbulent SVSs & - \\
\hline & & Vortex bursting & \\
\hline
\end{tabular}

SVSs: Secondary vortical structures; HVSs: Helical vorticity structures. 
the onset of the corresponding half-life times of averaged circulation and passive tracer concentration in the primary wake are close.

Vertical and horizontal extents of passive tracer are controlled by the environmental conditions. Moderately stable stratification supports detrainment and the rebound of the detrained fluid such that the tracer can be spread along significant vertical dimensions of more than six initial vortex separations. However, strong stratification inhibits wake vortex descent and vertical spreading of the passive tracer, instead the horizontal spreading achieve values of over seven initial vortex separations. The sensitivity of detrainment rates and tracer spreading on the initial tracer distributions within the wake appears to be low.

\section{ACKNOWLEDGMENTS}

The authors would like to thank Anthony P. Brown for fruitful discussions and providing information on vortex bursting and vortex funnel features. They also thank Sven Lüke for the kind provision of his contrail photo. In addition, valuable comments from Professor Ulrich Schumann are appreciated. The supply of computation time on supercomputers at the Deutsches Klimarechenzentrum (DKRZ), which was made available with a project of Dr. Andreas Dörnbrack, and at the Leibniz-Rechenzentrum (LRZ), which was under a project of Professor Robert Sausen, is greatly acknowledged. The current work has been conducted within the DLR Project Wetter und Fliegen (Weather and Flying).

${ }^{1}$ T. Gerz, F. Holzäpfel, and D. Darracq, "Commercial aircraft wake vortices,” Prog. Aerosp. Sci. 38, 181 (2002).

${ }^{2}$ P. Minnis, D. F. Young, L. Ngyuen, D. P. Garber, W. L. Smith Jr., and R. Palikonda, "Transformation of contrails into cirrus during SUCCESS," Geophys. Res. Lett. 25, 1157, doi:10.1029/97GL03314 (1998).

${ }^{3}$ U. Burkhardt, B. Kärcher, and U. Schumann, "Global modeling of the contrail and contrail cirrus climate impact,” Bull. Am. Meteorol. Soc. 91, 479 (2010).

${ }^{4}$ EUROCONTROL/STATFOR "Challenges to Growth 2008," Summary Report, November 2008, http://www.eurocontrol. int/statfor/public/standard_page/Challenges_to_Growth.html (cited 8 February 2012).

${ }^{5}$ C. P. van Dam, "The aerodynamics design of multi-element highlift systems for transport airplanes," Prog. Aerosp. Sci. 38, 101 (2002).

${ }^{6}$ T. Gerz, T. Dürbeck, and P. Konopka, "Transport and effective diffusion of aircraft emissions," J. Geophys. Res. 103, 25, doi:10.1029/98JD02282 (1998).

${ }^{7}$ T. Leweke and C. H. K. Williamson, "Cooperative elliptic instability of a vortex pair," J. Fluid Mech. 360, 85 (1998).

${ }^{8}$ K. K. Nomura, H. Tsutsui, D. Mahoney, and J. W. Rottman, "Short-wavelength instability and decay of a vortex pair in a stratified fluid," J. Fluid Mech. 553, 283 (2006).

${ }^{9}$ F. Laporte and A. Corjon, "Direct numerical simulation of the elliptic instability of a vortex pair," Phys. Fluids 12, 1016 (2000).

${ }^{10}$ D. Fabre and L. Jacquin, "Short-wave cooperative instabilities in representative aircraft vortices,” Phys. Fluids 16, 1366 (2004).

${ }^{11}$ J. Han, Y. Lin, D. G. Schowalter, and S. Pal Arya, "Large eddy simulation of aircraft wake vortices within homogeneous turbulence: Crow instability," AIAA J. 38, 292 (2000).

${ }^{12}$ F. H. Proctor, D. W. Hamilton, and J. Han, "Wake vortex transport and decay in ground effect: Vortex linking with the ground," in Proceedings of the 38th AIAA Aerospace Sciences Meeting, 10-13 January, Reno, Nevada (AIAA, 2000), Paper No. 2000-757.

${ }^{13}$ S. C. Crow, "Stability theory for a pair of trailing vortices," AIAA J. 8, 2172 (1970).

${ }^{14}$ T. Leweke and C. H.K. Williamson, "Experiments on long-wavelength instability and reconnection of a vortex pair," Phys. Fluids 23, 024101 (2011).

${ }^{15}$ F. Holzäpfel, T. Gerz, M. Frech, and A. Dörnbrack, "Wake vortices in convective boundary layer and their influence on following aircraft," J. Aircr. 37, 1001 (2000).

${ }^{16}$ F. Holzäpfel, T. Hofbauer, D. Darracq, H. Moet, F. Garnier, and C. F. Gago, "Analysis of wake vortex decay mechanisms in the atmosphere," Aerosp. Sci. Technol. 7, 263 (2003)

${ }^{17} \mathrm{H}$. Chevalier, "Flight test studies of the formation and dissipation of trailing vortices," J. Aircr. 10, 14 (1973).

${ }^{18}$ I. Tombach, "Observations of atmospheric effects on vortex wake behavior," J. Aircr. 10, 641 (1973).

${ }^{19}$ A. P. Brown, "On the specification of wake vortex encounter gust-fields from flight data," in Proceedings of the 2nd AIAA Atmospheric and Space Environments Conference, 2-5 August, Toronto, Ontario (AIAA, 2010), Paper No. 2010-7678.

${ }^{20}$ T. Sarpkaya and J. J. Daly, "Effect of ambient turbulence on trailing vortices," J. Aircr. 24, 399 (1987).

${ }^{21}$ H. Moet, F. Laporte, G. Chevalier, and T. Poinsot, "Wave propagation in vortices and vortex bursting," Phys. Fluids 17, 054109 (2005).

${ }^{22}$ P. R. Spalart, "Airplane trailing vortices," Annu. Rev. Fluid Mech. 30, 107 (1998).

${ }^{23}$ R. Sussmann and K. M. Gierens, "Lidar and numerical studies on the different evolution of vortex pair and secondary wake in young contrails,” J. Geophys. Res. 104, 2131, doi:10.1029/1998JD200034 (1999). 
${ }^{24}$ D. C. Lewellen and W. S. Lewellen, "The effects of aircraft wake dynamics on contrail development," J. Atmos. Sci. 58, 390 (2001).

${ }^{25}$ S. Unterstrasser and I. Sölch, "Study of contrail microphysics in the vortex phase with a Lagrangian particle tracking model," Atmos. Chem. Phys. 10, 10003 (2010).

${ }^{26}$ R. Paugam, R. Paoli, and D. Cariolle, "Influence of vortex dynamics and atmospheric turbulence on the early evolution of a contrail," Atmos. Chem. Phys. 10, 3922 (2010).

${ }^{27}$ D. D. Vicroy, J. Brandon, G. Greene, R. Rivers, G. Shah, E. Stewart, and R. Stuever, "Characterizing the hazard of a wake vortex encounter," in Proceedings of the 35th AIAA Aerospace Sciences Meeting, 6-9 January, Reno, Nevada (AIAA, 1997), Paper No. 1997-55.

${ }^{28}$ M. Manhart, "A zonal grid algorithm for DNS of turbulent boundary layer," Comput. Fluids 33, 435 (2004).

${ }^{29}$ P. R. Bannon, "Potential vorticity conservation, hydrostatic adjustment, and the anelastic approximation," J. Atmos. Sci. 52, 2302 (1995).

${ }^{30}$ T. Gerz and T. Ehret, "Wingtip vortices and exhaust jets during the jet regime of aircraft wakes," Aerosp. Sci. Technol. 1, 463 (1997).

${ }^{31}$ U. Schumann, P. Konopka, R. Baumann, R. Busen, T. Gerz, H. Schlager, P. Schulte, and H. Volkert, "Estimates of diffusion parameters of aircraft exhaust plumes near the tropopause from nitric oxide and turbulence measurements," J. Geophys. Res. 100, 14147, doi:10.1029/95JD01277 (1995).

${ }^{32}$ M. H. Kobayashi, "On a class of Páde finite volume methods," J. Comput. Phys. 156, 137 (1999).

${ }^{33}$ A. Hokpunna and M. Manhart, "Compact fourth-order finite volume method for numerical solutions of Navier-Stokes equations on staggered grids," J. Comput. Phys. 229, 7545 (2010).

${ }^{34}$ A. Hokpunna, "Compact fourth-order scheme for numerical simulations of Navier-Stokes equations," Ph.D. dissertation (Technische Universität München, Germany, 2009).

${ }^{35} \mathrm{C}$. W. Hirt and J. L. Cook, "Calculating three-dimensional flows around structures and over rough terrain," J. Comput. Phys. 10, 324 (1972).

${ }^{36}$ J. H. Williamson, "Low-storage Runge-Kutta schemes," J. Comput. Phys. 35, 48 (1980).

${ }^{37}$ C. Meneveau, T. S. Lund, and W. H. Cabot, "A Lagrangian dynamic subgrid-scale model of turbulence," J. Fluid Mech. 319, 353 (1996).

${ }^{38}$ S. Shen, F. Ding, J. Han, Y. Lin, S. P. Arya, and F. H. Proctor, "Numerical modeling studies of wake vortices: Real case simulations," in Proceedings of the 37th AIAA Aerospace Sciences Meeting, 11-14 January, Reno, Nevada (AIAA, 1999), Paper No. 1999-755.

${ }^{39}$ F. Holzäpfel, "Adjustment of subgrid-scale parameterizations to strong streamline curvature," AIAA J. 42, 1369 (2004).

${ }^{40}$ F. Holzäpfel, T. Gerz, and R. Baumann, "The turbulent decay of trailing vortex pairs in stably stratified environments," Aerosp. Sci. Technol. 5, 95 (2001).

${ }^{41}$ U. Schumann, H. Schlager, F. Arnold, R. Baumann, P. Haschberger, and O. Klemm, "Dilution of aircraft exhaust plumes at cruise altitudes," AAtmos. Environ. 32, 3097 (1998).

${ }^{42}$ R. Sussmann and K. M. Gierens, "Difference in early contrail evolution of two-engine versus four-engine aircraft: Lidar measurements and numerical simulations," J. Geophys. Res. 106, 4899, doi:10.1029/2000JD900533 (2001).

${ }^{43}$ I. Hennemann and F. Holzäpfel, "Large-eddy simulation of aircraft wake vortex deformation and topology," J. Aerosp. Eng., Part G 225, 1336 (2011).

${ }^{44}$ W. Béchara, C. Bailly, P. Lafon, and S. M. Candel, "Stochastic approach to noise modeling for free turbulent flows," AIAA J. 32, 455 (1994).

${ }^{45}$ D. K. Lilly, "Stratified turbulence and the mesoscale variability of the atmosphere," J. Atmos. Sci. 40, 749 (1983).

${ }^{46}$ F. Holzäpfel and T. Gerz, "Two-dimensional wake vortex physics in the stably stratified atmosphere," Aerosp. Sci. Technol. 3, 261 (1999).

${ }^{47}$ F. Holzäpfel, T. Gerz, F. Köpp, E. Stumpf, M. Harris, R. I. Young, and A. Dolfi-Bouteyre, "Strategies for circulation evaluation of aircraft wake vortices measured by lidar," J. Atmos. Oceanic Technol. 20, 1183 (2003).

${ }^{48}$ I. Hennemann and F. Holzäpfel, "Aircraft wake vortex curvature and resulting risk potential for following aircraft," CEAS 2009 European Air and Space Conference, Munchester, UK (2009).

${ }^{49}$ F. Holzäpfel, "Probabilistic two-phase wake vortex decay and transport model," J. Aircr. 40, 323 (2003).

${ }^{50}$ F. Holzäpfel, T. Misaka, and I. Hennemann, "Wake-vortex topology, circulation, and turbulent exchange processes," in Proceedings of the 2nd AIAA Atmospheric and Space Environments Conference, 2-5 August, Toronto, Ontario (AIAA, 2010), Paper No. 2010-7992.

${ }^{51}$ J. F. Garten, J. Werne, D. C. Fritts, and S. Arendt, "Direct numerical simulations of the Crow instability and subsequent vortex reconnection in a stratified fluid," J. Fluid Mech. 426, 1 (2001).

${ }^{52} \mathrm{~T}$. Birner, "Fine-scale structure of the extratropical tropopause region," J. Geophys. Res. 111, D04104, doi:10.1029/2005JD006301 (2006).

${ }^{53}$ J. M. Faddy and D. I. Pullin, "Flow structure in a model of aircraft trailing vortices," Phys. Fluids 17, 085106 (2005). 